\title{
An experimental comparison of collective choice procedures for excludable public goods
}

\author{
Sean Gailmard ${ }^{\mathrm{a}}$, Thomas R. Palfrey ${ }^{\mathrm{b}, *}$ \\ ${ }^{a}$ Department of Political Science, Northwestern University, United States \\ ${ }^{\mathrm{b}}$ Department of Politics and Economics, Princeton University, United States
}

Received 13 March 2002; received in revised form 28 December 2002; accepted 23 April 2003

Available online 14 November 2004

\begin{abstract}
This paper compares three collective choice procedures for the provision of excludable public goods under incomplete information. One, serial cost sharing (SCS), is budget balanced, individually rational, anonymous and strategy proof. The other two are "hybrid" procedures: voluntary cost sharing with proportional rebates (PCS) and with no rebates (NR). PCS satisfies all these properties except strategy proofness, and NR satisfies all the properties except for strategy proofness and budget balance. However, PCS and NR do not exclude any potential users, and they do not require equal cost shares, thereby overcoming the two main sources of inefficiency with SCS. We characterize the Bayesian Nash equilibria (BNE) of the hybrid mechanisms and conduct laboratory experiments to compare the performance of the three mechanisms. We find that PCS produces significantly more efficient allocations than either SCS or NR.

(C) 2004 Elsevier B.V. All rights reserved.
\end{abstract}

Keywords: Serial cost sharing; Proportional rebates; No rebates

\section{Introduction}

For the last several decades, economists and Social Scientists have grappled with the question of how to design mechanisms for the efficient production and cost sharing of public goods. Nearly all of the energy has been directed toward the case of pure,

* Corresponding author. Tel.: +1 609258 4031; fax: +1 6092588854.

E-mail addresses: gailmard@northwestern.edu (S. Gailmard), tpalfrey@princeton.edu (T.R. Palfrey). 
nonexcludable public goods. While the reasons for focusing on that kind of public good are historical and well rooted in the traditional literature, it is not at all clear that this is the most typical case in practice. Many public goods are excludable. Even the classic examples like lighthouses, parks and security can, in principle (and often do, in practice), exclude some users.

Recently, there has been an upsurge of interest in the design of mechanisms for excludable public goods. ${ }^{1}$ These mechanisms focus largely on cost sharing schemes, which assign different levels of usage to different consumers, and have a formula for assigning cost shares based on level of usage. The threat to exclude can serve to relax incentive constraints. But this comes with a cost, and this cost has been largely ignored in the literature. The very act of exclusion creates inefficiencies in a very direct way, which we call exclusionary inefficiency. Once a public good is provided, it is costless to allow extra users, so it is inefficient to exclude. ${ }^{2}$ A subset of the mechanisms for production and cost sharing of excludable public goods are mechanisms that exclude nobody. In mechanisms of this sort, there is no exclusionary inefficiency, so the only source of inefficiency is from free riding problems that result in underproduction of the public good.

In this paper, we report on experiments for the provision of excludable threshold public goods, and compare the performance of a widely acclaimed cost sharing mechanism, serial cost sharing, with two very simple and practical nonexclusionary rules, which are variants of a voluntary contributions mechanism. To keep the experiment as simple as possible, and to be able to compute Bayesian Nash equilibria (BNE) of the voluntary contributions mechanisms, we consider a discrete public good technology where, if a certain threshold of contributions is met, the good is produced and may be consumed at no extra cost to any subset of the group; the good is not produced at all if the threshold is not met. Consumers have private valuations of the public good and there is incomplete information about these preferences.

The basic question at issue here is whether the excellent incentive properties of the serial cost sharing (SCS) mechanism also imply good efficiency properties, relative to mechanisms that require ex post individual rationality. Since we are in a second best world, it does not make sense to compare serial cost sharing to the first best allocation, a comparison in which it would fare poorly. Instead, a natural benchmark is to compare serial cost sharing to mechanisms that are practical to implement and are widely observed. For this reason, we chose two variants on the voluntary contributions mechanism. In these mechanisms, individuals simply make pledges toward the production of the public good and the total production is simply the efficient production given the total amount of pledges. ${ }^{3}$ In our discrete environment, this means that the public good is produced if and only if the sum of the pledges exceeds the threshold, or cost of production. Mechanisms of this sort resemble fund

\footnotetext{
${ }^{1}$ See, for example, Moulin (1994), Deb and Razzolini (1999), Chen and Khoroshilov (2003), Chen (2003), Dorsey et al. (2002) and Norman (2000).

2 This is true for nonrival public goods. In case of crowding or other sorts of user externalities, the inefficiencies created by exclusion are less severe and exclusion can even be efficiency enhancing.

3 The mechanisms differ only in how they treat excess pledges.
} 
drives or capital campaigns used by private organizations and clubs; they represent the basic mechanism by which public goods are provided through charities or philanthropy, and are simple versions of auction mechanisms that have been designed for specific

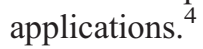

$\mathrm{SCS}^{5}$ is now quite familiar in the literature on excludable public goods (Moulin, 1994; Deb and Razzolini, 1999) and is related to the serial cost sharing mechanism for private goods (Moulin and Shenker, 1992). The mechanism is strategy proof, balanced and individually rational. The two nonexclusionary mechanisms are voluntary cost sharing with proportional rebates (PCS) and with no rebates (NR) of any contributions beyond the threshold. These are "hybrid" mechanisms. NR can be viewed as an anonymous, individually rational base mechanism, to which PCS adds budget balance. None of the three mechanisms are fully efficient.

We characterize the set of Bayesian Nash equilibria in PCS and NR, and establish several properties, including monotonicity of the bidding functions. Both mechanisms typically have multiple equilibria, in contrast to many auction mechanisms for public goods with independent private valuations. Each set includes some equilibrium allocation rules that are more efficient (and some that are less efficient) than the strategy proof allocation rule under SCS. The laboratory experiments provide information about the ability of these procedures to select "desirable" equilibria, and about how resulting collective choices compare on welfare grounds with those under SCS.

Our experiments find systematic differences between these procedures, in terms of both the individual and the collective behavior they induce. The dominant strategy equilibrium in SCS explains individual behavior very well. PCS induced significantly higher bidding than NR. Overbidding (i.e., bidding above value) was fairly common-more common than we anticipated - in PCS, but it was rarely observed in NR. Learning does not appear to have much effect on subject behavior within a given procedure, in the sense that there is only minimal evidence of trends in bidding behavior. An alternative hypothesis, risk aversion, is one possible explanation for the overbidding.

PCS extracts more consumer surplus than SCS and makes efficient decisions more often. Unlike SCS, PCS is not strategy proof, but it overcomes the two main sources of inefficiency in SCS: it never excludes any potential users of the public good and it allows unequal cost shares so high value users can subsidize low value ones. Furthermore, NR, which is neither strategy proof nor budget balanced, also overcomes these difficulties. The comparison between NR and SCS is more subtle since NR is not balanced. SCS delivers more consumer surplus and leads to ex post efficient decisions more often than NR. However, NR does substantially better when total rather than gross surplus (i.e., including overcontributions) is the welfare criterion. In fact, if the unrebated overcontributions are not subtracted in the surplus calculation, NR is comparable to SCS in extracting available surplus. Both are outperformed by PCS.

\footnotetext{
4 Examples abound. Andreoni $(1998,2001)$ provides many facts and illustrations. See Ferejohn et al. (1979) and Ferejohn et al. (1982) for a specific application to the sharing of programming costs across network affiliates.

5 More specifically, the direct serial mechanism to represent the serial formula (see Moulin, 1994). Our terminology follows Moulin's.
} 
There are three previous experimental studies of SCS, but all three examine serial cost sharing only for private good environments, where exclusion is not an issue. Chen (2003) and Chen and Khoroshilov (2003) investigate serial cost sharing and average cost pricing in complete information private good environments, and in environments with very limited information where subjects observe their own action and payoff, but not the game form or the other subjects' choices or payoffs. They focus on the learning dynamics under these mechanisms. Dorsey et al. (2002) have also studied SCS experimentally. In contrast to our results, they report that SCS performs relatively well on efficiency grounds, but this is because they examine a private goods environment rather than a public goods environment. The primary source of inefficiency in our experiment is underproduction, due to free riding, which does not present a problem in the private goods environment.

This paper is also related to the interdisciplinary body of experimental work on threshold or step-level public goods, where the level of the public good consumed is positive if and only if contributions exceed some threshold (e.g., Bagnoli and McKee, 1991; Isaac et al., 1988; Marwell and Ames, 1979, 1980; Palfrey and Rosenthal, 1991; Rapoport, 1985, 1987, 1988; Rapoport and Eshed-Levy, 1989; Rapoport and Suleiman, 1993). Unlike our experiments, however, these papers typically focus on variants of the voluntary contribution mechanism under complete information, and often on all-ornothing contributions. Suleiman and Rapoport (1992) study continuous contributions when return from public good is independent of total contributions (provided they exceed the threshold), an environment closely related to ours for PCS and NR. They focus (like Isaac et al., 1988) on the effect changes in the threshold have on contributions and the probability of public good provision. They find (again like Isaac et al., 1988) that higher thresholds lead to higher contributions but lower probability of provision. Cadsby and Maynes (1998) study continuous contributions under complete information; in one treatment, contributions may be refunded if the threshold is not met (similar to NR; see also the provision point mechanism in Bagnoli and McKee, 1991). This "money back guarantee" increases contributions relative to the case when contributions are lost even if the threshold is not met.

Dawes et al. (1986) examine all-or-nothing contribution and rebates. Consistent with some of our findings with finer grained contributions in PCS and NR, they find that rebates (their "no fear" condition) increase contributions. Marks and Croson (1998) study mechanisms like PCS and NR with continuous contributions under complete information, in addition to a mechanism where surplus contributions finance a continuous public good. They find that Nash outcomes are more common under the no rebate procedure and that rebates significantly affect the variance of contributions. Both of these mechanisms are analogous to auctions in private good environments. In fact, our public good environments are isomorphic to private good environments with fixed costs, zero marginal costs and no capacity constraint.

Auction-like procedures, similar to PCS and NR, have also been experimentally examined by Smith (1979a,b, 1980), Ferejohn et al. (1979) and Ferejohn et al. (1982). Smith compares the performance of institutional rules that vary individual incentives. In these "auction mechanisms", agents submit bids for how much they want of one public good or a menu of public projects and cost shares they will accept. If all agents agree on a 
quantity and cost share, that is the outcome; otherwise, the public good is not produced. In Ferejohn, Forsythe and Noll, and Ferejohn, Forsythe, Noll and Palfrey, groups had to select several public projects to produce and decide how to split costs; different members had different values and budgets. The collective choice procedures involved features of voting rules, auctions and rebates.

van Dijk and Grodzka (1992) study threshold public good provision with limited information about asymmetric endowments. They focus on subject evaluations of the equity of contributions. They do not study the effects of incomplete information in the game theoretic sense or try to control beliefs about the information asymmetry. Croson and Marks (1999) study the PPM (examined in Bagnoli and McKee, 1991 under complete information), an all-or-nothing contribution procedure, under incomplete information. They find no significant differences in the likelihood of successful provision under complete and incomplete information.

The rest of the paper is organized as follows. Section 2 defines the environment, equilibrium and some key properties of collective choice procedures. In Section 3, we develop and review some theoretical results on the mechanisms we test. In Section 4, we cover some properties of these mechanisms specific to our experimental parameters. In Section 5, we describe the design of the experiments. Section 6 discusses results from the experiments. Section 7 concludes.

\section{The model}

A group of individuals must decide whether to produce and how to share the cost of an excludable public good. There are $n$ players, and the output of the public good, $g$, is either 0 or 1 . The cost function is $C(1)=C>0$ and $C(0)=0$. The set of possible valuations for player $i$, denoted $V$, is a finite set of real numbers, and is the same for all players. We use $v_{i}$ to denote $i$ 's valuation and $v=\left(v_{i}, \ldots, v_{n}\right) \in V^{n}$ denotes a profile of valuations. Player $i$ knows $v_{i}$, but does not know $v_{j}$ for $j \neq i$. Values are independently and identically distributed across the players, according to the probability distribution function, $p$, which is common knowledge among the players. We assume that $\max \{V\}<C$, and that for some profile $v, \sum_{i} v_{i} \geqslant C$. The set of feasible outcomes is $A=\left\{g, s_{i}, \ldots, s_{n}, x_{1}, \ldots, x_{n} \mid g \in\{0,1\}\right.$, $\left.s_{i} \geqslant 0 \forall i, \sum_{i} s_{i} \geqslant C(g), 0 \leq x_{1} \leq g\right\}$ where $s_{i}$ is $i$ 's share of $C(g)$. Player $i$ 's utility of an outcome $a=\left(g, s_{i}, \ldots, s_{n}, x_{1}, \ldots, x_{n}\right)$, is given by $U_{i}(a)=v_{i} x_{1}-s_{i}$. We call $g$ the public good decision, $s_{i}$ is $i$ 's cost share and $x_{\mathrm{i}}$ is $i$ 's public good allocation.

A collective choice procedure is simply a mechanism or game form, $(B, f)$, where $B=B_{1} \times \cdots \times B_{n}$ is the message space and $f: B \rightarrow A$ is the outcome function. The outcome function $f$ has three components, $f=(G, S, X)$.

Definition 1. A direct mechanism is one where $B \equiv V$.

Definition 2. A strategy for player $i$ is a function $b_{i}: V_{i} \rightarrow B_{i}$. A strategy profile $b(v)=\left(b_{1}\left(v_{i}\right), \ldots, b_{n}\left(v_{n}\right)\right)$ is one strategy for each player.

Definition 3. The real valued function $b_{i}\left(v_{i}\right)$ is weakly monotone if $v_{i}>v_{i}^{\prime}$ implies $b_{i}\left(v_{i}\right) \geqslant b_{i}\left(v_{i}^{\prime}\right)$. It is strictly monotone if $v_{i}>v_{i}^{\prime}$ implies $b_{i}\left(v_{i}\right)>b_{i}\left(v_{i}^{\prime}\right)$. 
When player $i$ has valuation $v_{i}$, his payoff function in the game induced by the mechanism is:

$$
u_{i}(b(v), v)=v_{i} X_{i}(b(v))-S_{i}(b(v))
$$

Let $v_{-i}$ be all elements of $v$ besides $v_{i}$, and similarly for $b_{-i}\left(v_{-i}\right)$.

Definition 4. A pure strategy Bayesian Nash equilibrium is a strategy profile $b(\cdot)$ such that $b_{i}(\cdot) \in \arg \max _{b_{i}{ }^{\prime} \in B_{i}} \sum_{v_{-1}} p\left(v_{-i} \mid v_{i}\right) u_{i}\left(\left(b_{i}{ }^{\prime}, b_{-i}\left(v_{-i}\right)\right), v\right) \forall i$.

Definition 5. An equilibrium, $b$, is symmetric if for every $v^{\prime} \in V, b_{i}\left(v^{\prime}\right)=b_{j}\left(v^{\prime}\right)=b\left(v^{\prime}\right), \forall i, j$.

Definition 6. An equilibrium, $b$, is trivial if $G(b(v))=0$ for all $v$.

Definition 7. A strategy $b_{i}$ for player $i$ is ex post weakly dominated if there exists a $b_{i}^{\prime}$ such that for all value profiles $v, u_{i}\left(\left(b_{i}^{\prime}, b_{-i}\left(v_{-i}\right)\right), v\right) \geqslant u_{i}\left(\left(b_{i}, b_{-i}\left(v_{-i}\right)\right), v\right)$ for all $b_{-i}\left(v_{-i}\right)$ with strict inequality for some $b_{-i}\left(v_{-i}\right)$ and some $v$.

Thus, when $i$ knows all other values, $b_{i}$ is never better than, and sometimes strictly worse than, $b_{i}^{\prime}$, no matter what players other than $i$ do. Ex post weak domination implies weak domination at the interim stage, when $i$ knows $v_{i}$ but not $v_{-i}$ (as in the experiments), and at the ex ante stage, when $i$ knows neither her own value nor those of any other players, but only the prior on $v$.

Let $z^{T}$ be the components of a vector belonging to a set $T$.

Definition 8. A direct mechanism is ex post coalition strategy proof if for any $M \subseteq N$, any value profile $v \in \prod_{i=1}^{n} V$ and any profile of messages $\left(\hat{v}^{M}, \hat{v}^{M / N}\right) \in \prod_{i=1}^{n} V$, \#\{u $\left(\left(\hat{v}^{M}, \hat{v}^{M / N}\right), v\right)>u_{i}\left(\left(\hat{v}^{M}, \hat{v}^{M / N}\right), v\right)$ for some $\left.i \in M\right\}<\#\left\{u_{j}\left(\left(\hat{v}^{M}, \hat{v}^{M / N}\right), v\right)<u_{j}\left(\left(v^{M}, \hat{v}^{M / N}\right), v\right)\right.$ for some $j \in M\}$.

Thus, if any subset $M$ of $N$ (including the singleton $i$ ) could coordinate a deviation from the truth, more members of $M$ are worse off than better off, regardless of what $k \in M / N$ does.

Definition 9. A mechanism is ex post individually rational if $u_{i}(b(v), v) \geqslant 0 \forall v \in V, \forall i$.

Definition 10. A mechanism is anonymous if at any value profile $v$ such that $v_{i}=v_{j}$ and $v_{-i}=v_{-j}, u_{i}\left(\left(b_{i}\left(v_{i}\right), b_{-i}\left(v_{-i}\right)\right),\left(v_{i}, v_{-i}\right)\right)=u_{j}\left(\left(b\left(v_{j}\right), b_{-j}\left(v_{-j}\right)\right),\left(v_{j}, v_{-j}\right)\right) \forall i, j$.

Definition 11. A mechanism is budget balanced if $\sum_{i} S_{i}\left(b_{i}\right)=C, \forall b_{i}$.

One of our purposes is to compare the welfare properties of the various mechanisms. We define two measures here.

Definition 12. The consumer surplus of an outcome for the group $N$ is $\left(\sum_{i} v_{i} x_{1}-\sum_{i} s_{i}\right)$.

Definition 13. The total surplus of an outcome for the group $N$ is $\left(\sum_{i} v_{i} x_{i}-g C\right)$.

Both are measures of efficiency, and implicitly assume risk neutrality and transferable utility. The difference between the two measures is that the first measure considers excess cost shares to be deadweight loss, while the second does not. Therefore, the two surplus measures differ only if the budget is not balanced, that is, if $g C<\sum_{i} s_{i}$. Since the sum of the 
cost shares are equal for SCS and PCS, the two measures are the same in both mechanisms. For the NR mechanism, the second measure is always at least as great as the first measure.

\section{Properties of SCS, PCS and NR}

In this section, we review some properties of SCS. We also present some results on PCS and NR, and in particular properties of their sets of equilibria.

\subsection{Serial cost sharing}

With excludable public goods, there are several ways to express the serial cost sharing formula (Moulin, 1994). We focus on the direct serial mechanism associated with SCS. Represented in this way SCS has the attractive features of coalition strategy proofness, a strengthening of individual rationality, anonymity and budget balance.

Each agent is asked to report an element of $V$. Without loss of generality, suppose $\hat{v}_{1} \geqslant \hat{v}_{2} \geqslant \ldots \geqslant \hat{v}_{n}$ are the declared valuations. If $\sum_{i} \hat{v}_{i}<C$, the public good is not produced and all agents $i \in N$ pay $S_{i}(\hat{v})=0$. If $\sum_{i} \hat{v}_{i} \geqslant C$, and there is an integer $k$ such that $\hat{v}_{k} \geqslant C / k$, then the public good is produced. Furthermore, if $k^{*}$ is the largest integer such that $\hat{v}_{k} \geqslant C /$ $k$, then agents $j \in\left\{1,2, \ldots, k^{*}\right\}$ each consume the public good and all pay $S_{j}(\hat{v})=C / k^{*}$, while other agents $i \in\left\{k^{*}+1, \ldots, n\right\}$ do not consume the good and pay $S_{i}(\hat{v})=0$. If $\sum_{i} \hat{v}_{i} \geqslant C$ but there is not an integer $k$ such that $\hat{v}_{k} \geqslant C / k$, the good is not produced and all agents $i \in N$ pay $S_{i}(\hat{v})=0$. Note that an individual's payment is not affected by declarations of valuation higher than her own. This is an important component of the incentive scheme generated by this mechanism. Some important properties of SCS are listed in the following theorem.

Proposition 1 (Moulin, 1994, Theorem 2). SCS is ex post coalition strategy proof, ex post individually rational, budget balanced and anonymous.

This mechanism achieves budget balance in dominant strategies, so obviously it must not be efficient (cf. Green and Laffont, 1977) except in special uninteresting cases. In particular, to achieve incentive compatibility it exploits excludability of the public good: some agents are excluded from consumption of the public good in equilibrium. That is, for some agents and some profiles of reports, $x_{i}(b)<G(b)$. When $v_{i}>0$, this is ex post inefficient. A feature that leads indirectly to ex post inefficiency is that among the group members who consume the same amount of the public good, costs for that quantity of the good are shared equally. With a binary public good, then, all consumers must share costs equally. Thus, the mechanism cannot take advantage of a relatively high valuation to balance a relatively low one. For example, in a three person group, if the sum of all three valuations is higher than the cost, but none is higher than half the cost and one is less than a third of the cost, the SCS mechanism will not produce the good, even though by construction it would be efficient to do so. 


\subsection{Proportional cost sharing (PCS)}

In PCS, agents submit bids and share the cost of the public good only if the sum of all bids is greater than the cost of the good. This mechanism, like SCS, is individually rational, anonymous and balances the budget. However, it is not strategy proof. Nevertheless, it has two potential advantages over SCS. First, it never excludes anyone from consuming the public good, even though exclusion is feasible. Second, it allows for different agents to have different cost shares. This permits high valuation consumers to subsidize the consumption of low valuation consumers.

Agents submit real number bids from 0 to $C{ }^{6}$ If $\sum_{j} b_{j} \geqslant C$, the good is consumed by all agents and consumed by no agents otherwise. Agent $i$ 's cost share is

$$
s_{i}= \begin{cases}\frac{C b_{i}}{\sum_{j} b_{j}} & \text { if } \sum_{j} b_{j} \geqslant C \\ 0 & \text { if } \sum_{j} b_{j}<C\end{cases}
$$

Notice, therefore, that by construction, $\sum_{i} s_{i}=C$ if the good is produced, so PCS is budget balanced. Individual rationality and anonymity are similarly obvious.

There can be multiple Bayesian equilibria, and the set depends on the distribution of values and the production cost. Our first result is that none of the equilibria involve overbidding (bidding above valuation) and indeed this is dominated.

Proposition 2 (No overbidding). Any strategy in which $b_{i}\left(v_{i}\right)>v_{i}$ for some $v_{i}$ is ex post weakly dominated in the PCS mechanism.

Proof. Consider player $i$, suppose $b_{i}>v_{i}$, let $b_{i}^{\prime}=v_{i}$, and fix $b_{-i}\left(v_{-i}\right)$. First, if $\sum_{j \neq i} b_{j}\left(v_{j}\right)+b_{i} \geqslant C$ and $\sum_{j \neq i} b_{j}\left(v_{j}\right)+v_{i}<C$ at any value profile $v, i$ suffers a loss at that profile under $b_{i}: b_{i}=C-\sum_{j \neq i} b_{j}\left(v_{j}\right)$ implies $s_{i}(b)=b_{i}>v_{i}$, and when $\sum_{j=1}^{n} b_{j}\left(v_{j}\right)>C$, $\partial s_{i} / \partial b_{i}=C\left(\sum_{j \neq i} b_{j}\left(v_{j}\right)\right) /\left(\sum_{j=1}^{n} b_{j}\left(v_{j}\right)\right)^{2}$, which is strictly positive if $\sum_{j \neq i} b_{j}\left(v_{j}\right)>0$. If $\sum_{j \neq i} b_{j}\left(v_{j}\right)=0$, then $s_{i}=C>v_{i}$. But $i$ earns 0 payoff at such a profile with $b_{i}^{\prime}$. Second, if $\sum_{j \neq i} b_{j}\left(v_{j}\right)+b_{i} \geqslant C$ and $\sum_{j \neq i} b_{j}\left(v_{j}\right)+v_{i} \geqslant C$ at any value profile $v$, a bid of $b_{i}^{\prime}$ costs less than $b_{i}$, but does not affect the collective decision. Third, if $\sum_{j \neq i} b_{j}\left(v_{j}\right)+b_{i}<C$ at any value profile $v, i$ earns 0 payoff with both $b_{i}^{\prime}$ and $b_{i}$.

Let $P_{i}\left(b_{i}\right)=\operatorname{Pr}\left(\sum_{j \neq i} b_{j}\left(v_{j}\right) \geqslant C-b_{i}\right)$ denote the probability that when player $i$ bids $b_{i}$ the good is produced, given the bid functions of the other players. Let $S_{i}\left(b_{i}\right)=E_{v_{-i}}\left(s_{i}(b(v)) \mid b_{i}\right)$ denote $i$ 's expected cost share given a bid of $b_{i}$. We will use the following lemma. Note that, since valuations are strictly bounded above by $C$, the proposition above implies that $b_{i}<C$ for all $i$ in any BNE.

Lemma 1 (Cost share monotonicity). If $P_{i}\left(b_{i}\right)>0$ and $b_{i}<C, S_{i}\left(b_{i}\right)$ is strictly increasing at $b_{i}$.

Proof. $P_{i}\left(b_{i}\right)>0$ implies that the good is produced for at least some profiles. At all such profiles, $\partial s_{i} / \partial b_{i}>0$. At any profiles where the good is not produced, $\partial s_{i} / \partial b_{i}=0$.

\footnotetext{
${ }^{6}$ In the experiments agents submit integer bids from 0 to $C$. This does not affect the theoretical results.
} 
Proposition 3 (Bid monotonicity). Let $b^{*}(v)$ be a Bayesian Nash equilibrium bidding function in PCS. If $P_{i}\left(b^{*}(v)\right)>0$ for all $v>\min \{V\}$, then, for all $v_{i}, v_{j}, v_{i}>v_{j} \Rightarrow b^{*}\left(v_{i}\right) \geq b^{*}\left(v_{j}\right)$.

Proof. Consider two values $v_{i}$ and $v_{i}^{\prime}$ for player $i$, and some bid function such that $b_{i}\left(v_{i}\right)=b$ and $b_{i}\left(v_{i}^{\prime}\right)=b^{\prime}$. For $i$ to optimize, the following inequalities must hold:

$$
\begin{aligned}
& v_{i} P_{i}(b)-S_{i}(b) \geqslant v_{i} P_{i}\left(b^{\prime}\right)-S_{i}\left(b^{\prime}\right) \\
& v_{i}^{\prime} P_{i}\left(b^{\prime}\right)-S_{i}\left(b^{\prime}\right) \geqslant P_{i}(b)-S_{i}(b)
\end{aligned}
$$

Subtracting RHS of the second from LHS of the first and LHS of the second from RHS of the first yields

$$
\left(v_{i}-v_{i}^{\prime}\right)\left(P_{i}(b)-P_{i}\left(b^{\prime}\right)\right) \geqslant 0
$$

Then there are two cases to consider. First, $P_{i}(b)>P_{i}\left(b^{\prime}\right)$. Then if $v_{i}>v_{i}^{\prime}, b>b^{\prime}$ follows from the definition of a CDF. Second, $P_{i}(b)=P_{i}\left(b^{\prime}\right)>0$. Then suppose that $v_{i}>v_{i}^{\prime}$ but $b_{i}^{\prime}>b$; by Lemma 1 , this implies $S_{i}\left(b^{\prime}\right)>S_{i}(b)$, which implies $v_{i}^{\prime} P_{i}\left(b^{\prime}\right)-S_{i}\left(b^{\prime}\right)<v_{i}^{\prime} P_{i}(b)-S_{i}(b)$, which contradicts the assumption that $i$ optimizes.

\subsection{Cost sharing with NR}

Cost sharing with no rebates is the same as proportional cost sharing except excess contributions are not rebated. If the good is produced, then everyone simply pays their bid. Therefore, it is individually rational and anonymous. Like PCS, it never excludes anyone and it allows unequal cost shares, so high value users can subsidize low value users.

Formally, each agent $i$ submits a real number bid, $b_{i}$, from 0 to $C$. If $\sum_{j} b_{j} \geqslant C$, the good is consumed by all agents and consumed by no agents otherwise. Agent $i$ 's cost share is

$$
s_{i}= \begin{cases}b_{i} & \text { if } \quad \sum_{j} b_{j} \geqslant C \\ 0 & \text { if } \quad \sum_{j} b_{j}<C\end{cases}
$$

Therefore, if the good is produced, each agent simply pays its bid.

The equilibrium set for NR bears some similarities to that of PCS.

Proposition 4 (No overbidding). Any strategy in which $b_{i}\left(v_{i}\right)>v_{i}$ for some $v_{i}$ is ex post weakly dominated in the NR mechanism.

Proposition 5 (Bid monotonicity). Let $b^{*}(v)$ be a Bayesian Nash equilibrium bidding function in NR. If $P_{i}\left(b^{*}(v)\right)>0$ for all $v>\min \{V\}$, then, for all $v_{i}, v_{j}, v_{i}>v_{j} \Rightarrow b^{*}\left(v_{i}\right) \geq b^{*}\left(v_{j}\right)$.

The proofs of these two propositions are the same as for the analogous propositions in the previous section. Ex post weak dominance only required a $s_{i}\left(b_{i}\right)$ such that $\partial s_{i} / \partial b_{i}>0$ when $\sum_{j} b_{j} \geqslant C$ and $\sum_{j \neq i} b_{j}>0$, and Lemma 1 required for weak monotonicity is also true for NR. 


\section{Experimental parameters}

In our laboratory experiments, we used three person groups, with cost $C=102$ and each $v_{i}$ is independently drawn from a uniform distribution over a set of three possible values, $v_{i}=\{29,45,90\}, i=1,2,3$. The valuations were chosen in a way that allows the possibility of systematically different equilibrium behavior across the three mechanisms we wish to compare. Additional details of the experimental design are given in the next section. Let $\langle A, B, C\rangle$ denote the profile of values $A, B, C$ and all its permutations.

\subsection{Equilibria in SCS}

SCS has multiple equilibria under our parameters. All but one of these are trivial and use dominated strategies. There is a unique equilibrium in dominant strategies. For example, if all agents but one always claim to have $v=29$, the remaining agent cannot gain by claiming otherwise. The unique nontrivial SCS equilibrium entails truthful revelation by each bidder. It will produce the public good for at least some group members in the profiles $\langle 90,90,90\rangle,\langle 90,90,45\rangle,\langle 90,45,45\rangle,\langle 45,45,45\rangle$ and $\langle 90,90,29\rangle$. Only the last of these involves exclusion, and hence fails to realize all available surplus in the profile. The unique dominant strategy equilibrium makes the efficient decision (in the sense of producing if and only if the sum of values is at least 102) in 12 of 27 equally likely value profiles. Three of these 12 involve exclusion, so in 9 of 27 profiles, the weakly dominant SCS equilibrium is fully efficient.

If $v_{i} \in[2 C / 3, C], v_{2} \in[C / 3, C / 2]$ and $v_{3} \in[0, C / 3]$ where $v_{i}$ is the $i$ th highest value, the good is never produced even though it is always efficient to do so. Only one person is willing to share up to $1 / 2$ the cost and only two people are willing to share up to $1 / 3$. In the experiment, all three group members have an equal chance of drawing a value from one of these three regions. All three possible values used in the experiment are near the upper bound of their respective intervals, making the size of the lost surplus relatively large, in the probability $2 / 9$ event that each one of these regions is represented in a given group.

\subsection{Equilibria in PCS and NR}

Consider a symmetric bid function $b=\left\{b^{L}, b^{M}, b^{H}\right\}$ where $v=29$ bids $b^{L}, v=45$ bids $b^{M}$ and $v=90$ bids $b^{H}$. The following propositions are useful for calculating the set of equilibria for our experimental parameters. The first proposition states that the bids in nontrivial equilibria are bounded away from zero for all types in PCS and for all except the lowest demand type in NR. The third proposition states that, for both mechanisms, players never make a positive bid that would surely leave money on the table. In other words, if some type bids $b^{t}>0$ in equilibrium, then there must exist some pair of types of the other players, say $t^{\prime}$ and $t^{\prime \prime}$, such that $b^{t}+b^{t^{\prime}}+b^{t^{\prime \prime}}=102$. Proofs are given in Appendix B.

Proposition 6. In $P C S, P\left(b^{L}\right), P\left(b^{M}\right)$ and $P\left(b^{H}\right)$ are strictly positive in any nontrivial symmetric equilibrium. 
Proposition 7. In $N R, P\left(b^{M}\right)$ and $P\left(b^{H}\right)$ are strictly positive in any nontrivial symmetric equilibrium.

Proposition 8. Consider a symmetric profile of bid functions $b=\left\{b^{L}, b^{M}, b^{H}\right\}$ in PCS or $N R$. If player $i$ with value $v_{i}$ has any interim profitable deviation from $b^{v}$, then one of the following deviations is profitable: $d=0$ or $d=102-x-y$ for some $x, y \in b$.

Given that the experiment permitted only integer valued bids, we restrict attention in PCS and NR to equilibria in integer valued bid functions. We further restricted attention to pure strategy (and symmetric) equilibria. ${ }^{7}$ Given the restriction to integervalued bid functions, a numerical search for equilibria was most efficient. To compute nontrivial equilibria, for each weakly monotone, positive, integer valued, pure bid function with no overbidding, we checked each possible test deviation identified in Proposition 8.

In addition, the set of trivial symmetric pure strategy BNE under our experimental parameters are given by bid functions:

$$
b_{i}=\left\{\begin{array}{cl}
l & \text { if } v_{i}=29 \\
m & \text { if } v_{i}=45 \\
h & \text { if } v_{i}=90
\end{array}\right.
$$

where $0 \leqslant l \leqslant 6,0 \leqslant m \leqslant 6$ and $0 \leqslant h \leqslant 6$.

Under PCS, the nontrivial equilibria can be classified into four efficiency categories, as measured by total surplus. Within each category, the set of profiles at which the good is produced is the same. The most efficient group contains four equilibria, ${ }^{8}$ all of which involve some pooling. There are 17 equilibria in the second most efficient class, and 40 equilibria fall into the third most efficient group. The multiplicity of equilibria in a given category simply corresponds to different equilibrium cost shares for the same allocations. The fourth category produces for exactly the same set of profiles as in SCS, and includes the least efficient (nontrivial) PCS equilibria. The set of all symmetric PCS equilibria is graphed in Fig. 1.

For NR (Fig. 2), 35 equilibria are nontrivial; only 4 of these ${ }^{9}$ are more efficient than the undominated SCS equilibrium. ${ }^{10}$

Table 1 summarizes the equilibria for the three mechanisms, by identifying the type profiles for which the public good is provided (Table 1).

\subsection{Ex post efficiency of equilibria in SCS, PCS and NR}

From the derivation above, with our parameters the most efficient NR equilibrium is as efficient as the second most efficient PCS equilibrium. The most efficient and second most

\footnotetext{
7 This only restricts the class of equilibria we consider. All equilibria we find are equilibria with respect to the set of all integer valued bid functions.Focusing on symmetric equilibria for experimental purposes is not limiting, since subject matching precluded coordination on asymmetric equilibria.

$8\{21,21,60\},\{22,22,58\},\{23,23,56\}$ and $\{24,24,54\}$, where $\{X, Y, Z\}$ denotes the bid of type 29,45 and 90 , respectively.

$9\{23,34,45\},\{24,34,44\},\{25,35,42\}$ and $\{26,35,41\}$.

10 Equilibrium sets in PCS and NR are strictly non-nested.
} 


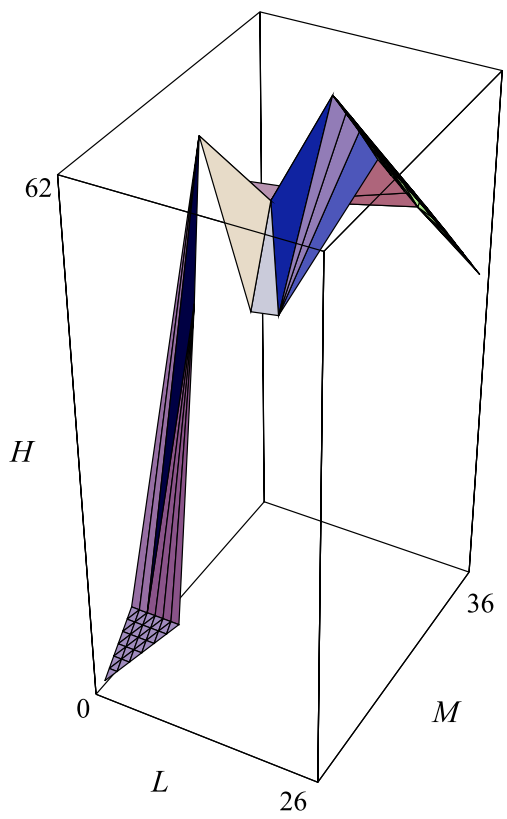

Fig. 1. Equilibrium set, proportional cost sharing mechanism.

efficient equilibrium under PCS produces higher expected surplus than the SCS dominant strategy equilibrium. The remaining 31 nontrivial, integer valued, symmetric, pure BNE in NR are strictly less efficient than the SCS equilibrium.

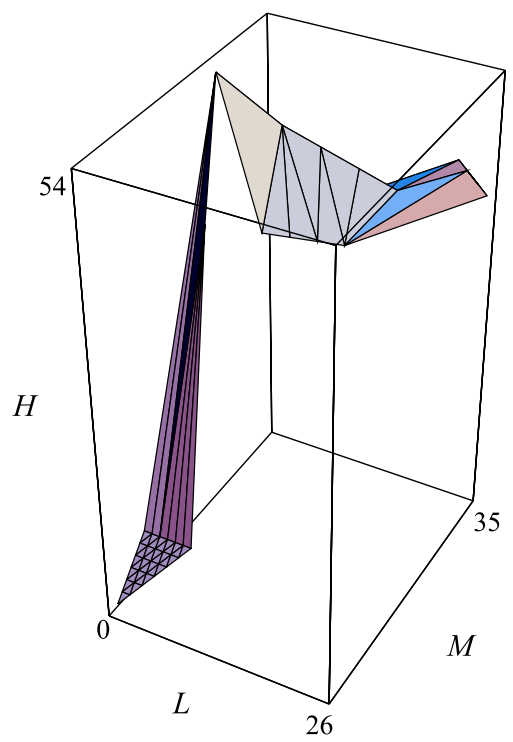

Fig. 2. Equilibrium set, no rebates mechanism. 
Table 1

Production decisions at each profile, by equilibrium class

\begin{tabular}{llllll}
\hline Profile & Equilibrium & & & & \\
\cline { 2 - 6 } & PCS-1 & PCS-2 & PCS-3 & PCS-4 (NR) & SCS \\
\hline$\langle L, L, L\rangle$ & & & & & \\
$\langle L, L, M\rangle$ & & & & $\mathrm{X}$ & $\mathrm{X}$ \\
$\langle L, M, M\rangle$ & & $\mathrm{X}$ & & \\
$\langle M, M, M\rangle$ & $\mathrm{X}$ & $\mathrm{X}$ & $\mathrm{X}$ & & $\mathrm{X}$ \\
$\langle L, L, H\rangle$ & $\mathrm{X}$ & $\mathrm{X}$ & $\mathrm{X}$ & $\mathrm{X}$ & $\mathrm{X}$ \\
$\langle L, M, H\rangle$ & $\mathrm{X}$ & $\mathrm{X}$ & $\mathrm{X}$ & $\mathrm{X}$ & $\mathrm{X}$ \\
$\langle M, M, H\rangle$ & $\mathrm{X}$ & $\mathrm{X}$ & $\mathrm{X}$ & $\mathrm{X}$ & $\mathrm{X}$ \\
$\langle L, H, H\rangle$ & $\mathrm{X}$ & $\mathrm{X}$ & $\mathrm{X}$ & $\mathrm{X}$ & \\
$\langle M, H, H\rangle$ & & & & & \\
$\langle H, H, H\rangle$ & & & & & \\
\hline
\end{tabular}

"PCS $k$ " denotes the $k$ th most efficient group of PCS equilibria. X denotes production.

a Denotes exclusion.

\subsection{Ex ante efficiency of equilibria in SCS, PCS and NR}

An allocation rule is ex ante efficient and individually rational if there does not exist another individually rational and feasible allocation rule that is a Bayesian equilibrium of some mechanism that ex ante Pareto dominates it. From results in Ledyard and Palfrey (1999a), ex ante efficiency implies no exclusion in our experimental environment. Full characterization of interim efficient allocation rules, of which ex ante efficient allocation rules are a special case, can be found in Ledyard and Palfrey (1999a).

In our environments, ex ante efficiency entails production of the public good at all profiles except $\langle 29,29,29\rangle$. We show that none of the three rules we study here are ex ante efficient. However, the departure from first best efficiency is quite small in the second best mechanism. Further, the best equilibria of PCS are $95.02 \%$ ex ante efficient, the best equilibria from NR are $88.79 \%$ ex ante efficient, and the dominant strategy SCS equilibrium is $61.57 \%$ ex ante efficient.

Proposition 9. No allocation rule is ex ante efficient.

Proof. The proof relies on no distortion at the top and binding adjacent incentive constraints. Let $t_{v}$ be the expected payment of type $v$. Conditional on reporting $v=29$, the ex ante efficient probability of consumption is $8 / 9$, so $v=45$ 's incentive constraint is $(45-29) 8 / 9 \leqslant 45-t_{45}$, or $t_{45} \leqslant 30.7778$. Then, $v=90$ 's incentive constraint is $90-30.7778 \leqslant 90-t_{90}$ or $t_{90} \leqslant 30.7778$. But feasibility requires $8 / 9(29)+30.7778+t_{90} \geqslant 26 / 27(102)$ or $t_{90} \geqslant 41.6667$. Thus, feasibility and incentive compatibility cannot both be satisfied.

Under our parameters, the best a welfare maximizing principal can do subject to incentive compatibility, individual rationality and feasibility is produce at all profiles except $\langle 29,29,29\rangle$ and $\langle 29,29,45\rangle$. This is implementable according to the profilecontingent payments in Table 2 .

A principal could present the agents with this table and they would willingly report their valuations. The expected taxes satisfy incentive compatibility and individual 
Table 2

Profile-contingent payments in the second-best allocation rule

\begin{tabular}{lrrr}
\hline Payments & \multicolumn{2}{c}{} \\
\hline Other types & 29 & 45 & 90 \\
\cline { 2 - 4 } & 0 & 0.0 & 44.0 \\
29,29 & 0 & 36.5 & 41.6 \\
29,45 & 29 & 31.4 & 36.5 \\
29,90 & 0 & 36.5 & 41.6 \\
45,29 & 29 & 34.0 & 39.9 \\
45,45 & 29 & 31.4 & 39.9 \\
45,90 & 29 & 31.4 & 36.5 \\
90,29 & 29 & 31.4 & 39.9 \\
90,45 & 29 & 31.4 & 34 \\
90,90 & & & \\
\hline
\end{tabular}

rationality: $t_{29}=2 / 3(29), t_{45}=29.333$ and $t_{90}=39.333$. They also satisfy feasibility: $t_{29}+t_{45}+t_{90} \geqslant 23 / 27(102)$.

\subsection{Hypotheses}

Based on the previous equilibrium results, we formulated the following hypotheses (Table 3). The first three deal with individual-level behavior; the second three cover collective welfare properties. All are based on the assumption of equilibrium behavior (Table 3).

We conjecture Hypothesis 2 because relative to NR, PCS lowers the cost of high bids. NR has equilibria where subjects bid more than PCS, but we had no reason to believe such equilibria would be selected.

In view of the multiple equilibria in PCS, Hypothesis 4 and the assumption of equilibrium behavior implies a statement about equilibrium selection. Given the large number to choose from, we believed subjects would be able to coordinate on PCS equilibria more efficient than the undominated SCS equilibrium. We wish to avoid Pareto

Table 3

\section{(a) Individual-level hypotheses}

$\mathrm{H} 1$

$\mathrm{H} 2$

$\mathrm{H} 3$

(b) Collective-level hypotheses

\begin{tabular}{ll}
\hline $\mathrm{H} 4 \mathrm{a}$ & PCS will extract more available consumer surplus than SCS \\
$\mathrm{H} 4 \mathrm{~b}$ & PCS will make efficient decisions more often than SCS \\
$\mathrm{H} 5 \mathrm{a}$ & NR will extract more available consumer surplus than SCS \\
$\mathrm{H} 5 \mathrm{~b}$ & NR will make efficient decisions more often than SCS \\
$\mathrm{H} 6 \mathrm{a}$ & PCS will extract more available surplus than NR \\
$\mathrm{H} 6 \mathrm{~b}$ & PCS will make efficient decisions more often than NR \\
\hline
\end{tabular}

\section{Subjects will reveal their types truthfully in SCS}

Bids in PCS will be higher than bids in NR

Bidding above value will not occur in PCS or NR

PCS will make efficient decisions more often than NR 
Table 4

Experiment design

\begin{tabular}{lllcl}
\hline Session no. & 1st treatment & 2nd treatment & \# subjects & Subj. pool \\
\hline 1 & SCS & PCS & 12 & CIT \\
2 & SCS & PCS & 9 & PCC \\
3 & SCS & PCS & 9 & PCC \\
4 & PCS & NR & 9 & PCC \\
5 & NR & PCS & 9 & PCC \\
6 & PCS & NR & 9 & PCC \\
7 & NR & PCS & 9 & PCC \\
\hline
\end{tabular}

efficiency as a precise equilibrium selection device, while still maintaining that higher surplus equilibria should be "focal".

Given the equilibrium sets for our parameters, we conjectured that NR may not outperform SCS on efficiency grounds, since NR has fewer equilibria that are more efficient than the SCS equilibrium. Therefore, we conjecture that Hypothesis 5 is less likely to be borne out in the data, compared to Hypothesis 4 .

NR's most efficient equilibria are as efficient, in terms of likelihood of production and surplus extraction, as PCS's second-most efficient equilibria. Hypothesis 6 implies that NR is not systematically better than PCS at inducing coordination on its most efficient equilibria.

\section{Experimental design}

To test our hypotheses, we conducted seven sessions of the experiment in the Social Science Experimental Laboratory at Caltech. Each session had two parts, with the two parts using different mechanisms and each part repeated over 10 rounds. Subjects for most sessions were students at Pasadena City College (PCC), but for one session, the first SCSPCS session, they were undergraduates at Caltech (CIT). All PCC sessions had nine subjects. The Caltech session had 12 subjects. Subjects participated in each mechanism for 10 rounds, where a round consisted of one decision by each subject and a resulting collective decision on public good consumption for the group. This information is summarized in Table 4.

Instructions were read aloud to the subjects at the beginning of the experiment to ensure public knowledge of the procedures. In addition, for each mechanism there were two practice rounds ${ }^{11}$ to familiarize subjects with the procedures. At the beginning of each round, subjects were randomly matched into groups of three as follows: numbered ping pong balls were placed in a box and shuffled in plain sight of the subjects. Three ping pong balls were drawn from the box and the subjects whose numbers were on the balls constituted group 1. This process was repeated (without replacement) to draw the

\footnotetext{
11 An exception to this was when NR run second, where only one practice round with that mechanism was used.
} 
Table 5

Messages, collective decisions and cost shares SCS

\begin{tabular}{llllll}
\hline$\# H$ messages & $\# M$ messages & $\# L$ messages & Produce? & \# Users & Cost share \\
\hline 3 & 0 & 0 & yes & 3 & 34 \\
2 & 1 & 0 & yes & 3 & 34 \\
1 & 2 & 0 & yes & 3 & 34 \\
0 & 3 & 0 & yes & 3 & 34 \\
2 & 0 & 1 & yes & 2 & 51 \\
1 & 1 & 1 & no & 0 & - \\
1 & 0 & 2 & no & 0 & - \\
0 & 2 & 1 & no & 0 & - \\
0 & 1 & 2 & no & 0 & - \\
0 & 0 & 3 & no & 0 & \\
\hline
\end{tabular}

remaining groups in each round. Subjects did not know the other members of their group in any round; they only knew that they were randomly re-matched after every decision.

An experimenter then went from subject to subject to have each one independently throw a six-sided die to determine his or her valuation for the round. ${ }^{12}$ A roll of 1 or 2 meant $v_{i}=29$, a roll of 3 or 4 meant $v_{i}=45$ and 5 or 6 meant $v_{i}=90$. Subjects each then decided what to bid for the round and recorded the decision on the decision/ record sheet. In SCS, subjects could bid either low, medium or high, meaning a willingness to share none, $1 / 3$ or $1 / 2$ of the cost $C=102$, respectively. ${ }^{13}$ In PCS and $\mathrm{NR}$, subjects could bid any integer between 0 and the cost $C=102$. When all decisions were made, an experimenter collected the sheets. For each group, decisions on whether the public good was produced and who consumed it were made according to the rules of the mechanism being played. For our SCS experiments, these are shown in Table 5.

For PCS and NR, the rules were the ones described in Section 2 above. The experimenter returned the sheets to the subjects, and the next round proceeded in this same way. More details are available in the instructions (see Appendix A).

\section{Results}

In this section, we discuss the results of our experiments, both in terms of the decision making behavior within each mechanism and in terms of the performance of each mechanism on welfare grounds.

\footnotetext{
12 The subjects were separated by privacy partitions, so that the outcome of their roll of the die was not observable to any other subject.

13 There are several ways one could implement the SCS mechanism in our environment. For example, one could use a direct mechanism that elicits reports of valuations (bids), and then convert these reports into one of the three categories, L, M or $\mathrm{H}$, in order to make an production and allocation decision. This would have added an unnecessary layer of complexity, so we chose the simpler method.
} 


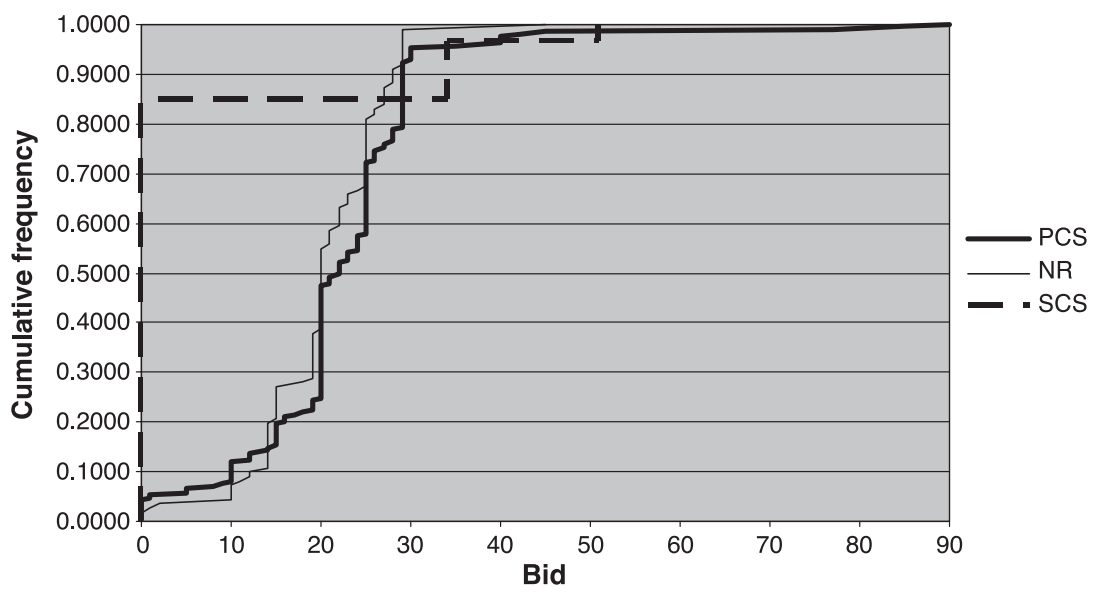

Fig. 3. Bid CDFs, value $=29$, by mechanism.

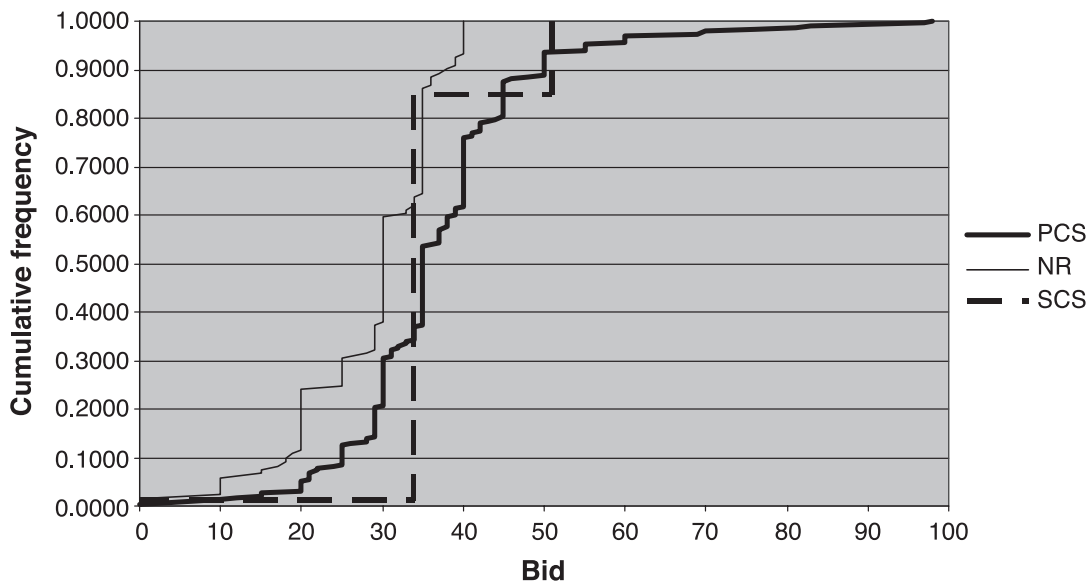

Fig. 4. Bid CDFs, value $=45$, by mechanism.

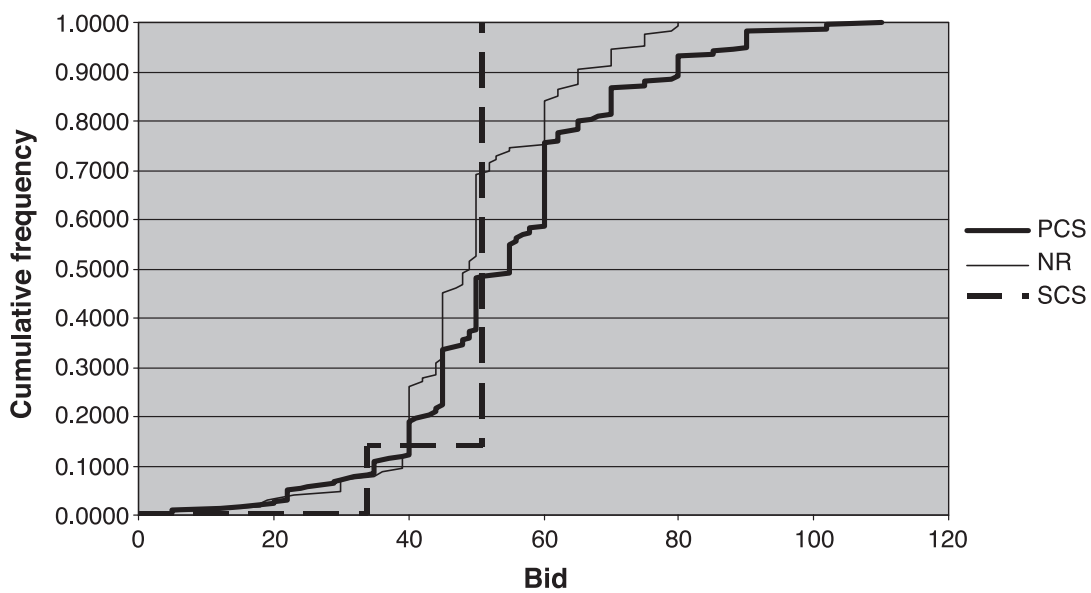

Fig. 5. Bid CDFs, value $=90$, by mechanism. 


\subsection{Individuals: bidding behavior}

Figs. 3-5 display the CDFs of bids under each mechanism for $v=29,45$ and 90, respectively. The CDFs for SCS were computed by mapping a message of $L, M$ or $H$ in that mechanism to a bid of 0,34 and 51, respectively. Since these are the implied cost sharing offers for each message in that mechanism, this is the most straightforward way to compare SCS directly with NR and PCS. ${ }^{14}$

These CDFs reveal important differences in behavior in these procedures. For $v=29$, both NR and PCS induce higher bidding than SCS. Over $85 \%$ of bidders in SCS were willing to share none of the cost (rather than one-half or one-third), while in PCS and NR virtually all bidders were willing to share some of the cost. In PCS, approximately $80 \%$ of bidders and in NR 70\% of bidders were willing to share 20 units or more of the cost when $v=29$. For $v=45$, only about $15 \%$ of bidders in SCS are willing to share more than onethird of the cost or 34 units; in PCS about $65 \%$ and in NR about $35 \%$ were willing to share more than one-third (though for NR only about $15 \%$ were willing to share more than 35 units). In SCS, no bidder can share more than one-half of the cost and, for $v=90$ in SCS, $86 \%$ of bidders are willing to share this much. About $50 \%$ of bidders in PCS and about $30 \%$ in NR are willing to share more than one-half the cost.

\subsubsection{Bidding behavior in SCS: a quantal response explanation for disequilibrium bids}

The weakly dominant equilibrium in SCS does a good job of explaining subject behavior, in accordance with Hypothesis 1. Table 6 presents the empirical proportions of time a subject made each bid, conditional on value. The figures are aggregated over all three sessions of SCS. Thus, conditional on $v=29,85.1 \%$ of bids were truthful.

Nevertheless, Nash equilibrium (weakly dominant or otherwise) does leave some behavior unexplained in SCS. A model of equilibrium errors may add some explanatory power. Quantal response equilibrium (McKelvey and Palfrey, 1995, 1996, 1998) is a statistical version of Nash equilibrium, in which players sometimes choose suboptimal strategies. The likelihood of choosing a strategy is monotonic and continuous in the expected utility of the strategy. Therefore, the theory predicts that low-cost errors are more likely to occur than high-cost errors. Table 7 lists cost of deviating from the dominant Nash equilibrium (i.e., larger positive numbers imply more costly behavior), given the empirical bidding frequencies from Table 6.

Thus, for 29 bidders in the SCS mechanism, the optimal strategy is to announce $L$, while announcing $M$ is a low cost error and $H$ is a high cost error. For the 45 bidders, announcing $H$ is the low cost error and, for 90 bidders, announcing $M$ is the low cost error. This is consistent with the data. Specifically, for all valuations, low-cost errors are observed more frequently than high-cost errors (Table 6).

\subsubsection{Comparing bids under PCS and NR}

It is evident from Figs. 3-5 that, for each value, NR tends to lower bids relative to PCS, though this effect looks weaker for $v=29$. Kolmogorov-Smirnov tests (two-tailed) for

\footnotetext{
${ }^{14}$ Because any 1 to 1 correspondence from messages to bids can be used, it also makes sense to speak of "truthful" bids in SCS.
} 
Table 6

Empirical SCS bids, proportions

\begin{tabular}{llll}
\hline Bid/value & 29 & 45 & 90 \\
\hline$L$ & 0.851 & 0.010 & 0.000 \\
$M$ & 0.117 & 0.837 & 0.139 \\
$H$ & 0.032 & 0.153 & 0.861 \\
\hline
\end{tabular}

difference in distribution support these interpretations and, generally, Hypothesis 2. For $v=45$ and 90, the differences due to the mechanism are large and significant. For $v=29$, the difference is insignificant at the $\alpha=0.10$ level. Table 8 displays the observed max $\left|F_{\mathrm{PCS}}(b)-F_{\mathrm{NR}}(b)\right|$ for each value (where $F_{A}(b)$ is the CDF for the given value of mechanism $A$ ) and the critical values for $\alpha=0.10$ and 0.001 (see Siegel, 1956, Table M).

Higher bidding in PCS than NR is also reflected in overbidding as a proportion of all bids, conditional on value, where overbidding refers to bidding above value, a dominated action. While it is dominated, it is a less costly mistake under PCS than under NR. Empirically overbidding was much more common under PCS: in NR, it happened only two times in 360 decisions, supporting Hypothesis 3 for NR. In PCS, it happened 52 times in 660 decisions. This is common enough to lead to rejection of Hypothesis 3 for PCS. Table 9 displays results on bids above value (aggregated over all sessions for each mechanism).

Table 10 displays the median bids by value in each mechanism (aggregated over all sessions for each mechanism), for a given group of rounds.

For all three values, and in both early and later rounds, the median bid under PCS exceeds that under NR.

\subsubsection{Bidding behavior in NR and PCS and equilibrium predictions}

If all subjects used these median bids for each mechanism, PCS would produce the public good for profiles $\langle 90,90,90\rangle,\langle 90,90,45\rangle,\langle 90,90,29\rangle,\langle 90,45,45\rangle,\langle 90,45$, $29\rangle$ and $\langle 45,45,45\rangle$. NR would produce for $\langle 90,90,90\rangle,\langle 90,90,45\rangle,\langle 90,90,29\rangle$ and $\langle 90,45,45\rangle$.

The median bids suggest that in aggregate, subjects in PCS are not playing the most efficient symmetric, pure BNE. Since there are so many equilibria, it is difficult to rule out equilibrium as an explanation for aggregate bidding behavior. By a least squares criterion, the symmetric, pure BNE that best matches the empirical medians is $\{19,31,52\}$, but this exhibits different production behavior: it produces for all the same profiles as the median except $\langle 45,45,45\rangle$. In the set of symmetric, pure BNE that produce for the same profiles as the median bids, $\{19,34,49\}$ has the best fit by least squares.

For NR, the symmetric, pure BNE that best matched the aggregate empirical behavior by least squares is $\{23,34,45\}$, which is one of the most efficient for that mechanism. Yet

Table 7

Expected cost of deviating from expected bid function, based on empirical bid frequencies

\begin{tabular}{lrrr}
\hline $\mathrm{Bid} /$ value & \multicolumn{1}{c}{29} & \multicolumn{1}{c}{45} & 90 \\
\hline$L$ & -0.52 & 5.35 & 31.83 \\
$M$ & 2.02 & -0.24 & 3.36 \\
$H$ & 6.43 & 0.96 & -0.54 \\
\hline
\end{tabular}


Table 8

Kolmogorov-Smirnov test statistics and critical values

\begin{tabular}{lccr}
\hline Value & 29 & 45 & 90 \\
\hline $\max \left|F_{\mathrm{PCS}}(b)-F_{\mathrm{NR}}(b)\right|$ & 0.1301 & 0.3438 & 0.2404 \\
Critical, $\alpha=0.10$ & 0.1435 & 0.1360 & 0.1363 \\
Critical, $\alpha=0.001$ & 0.2294 & 0.2174 & 0.2179 \\
$n$ 's, PCS & 207 & 236 & 217 \\
$n$ 's, NR & 111 & 122 & 127 \\
\hline
\end{tabular}

under the median bids for NR, aggregate outcomes in that mechanism are not as efficient as in this equilibrium. Among the symmetric, pure BNE where production behavior matched that under the medians, $\{18,30,42\}$ has the best fit by least squares.

In neither mechanism do subjects converge to trivial equilibria. Subjects rarely submit 0 bids. Table 11 shows the proportion of 0 bids.

\subsection{Other explanations for deviations from equilibrium behavior}

We consider two possible explanations that could account, at least in part, for the deviations of observed behavior from theory. The first explanation is simply that the design did not permit perfect control over preferences. We explore one possible factor, risk aversion, which could lead to overbidding. ${ }^{15}$ Second we consider the possibility that initially behavior was out of equilibrium and was gradually converging to equilibrium. The analysis above aggregates behavior across both early rounds and later rounds, implicitly assuming that there are no significant time trends in the data, due to learning. While we do not propose or estimate a specific learning model, we do report evidence that the differences in subject behavior between early rounds and later rounds is of minor consequence.

\subsubsection{Risk aversion as a possible explanation of overbidding}

As noted in the last section, bidding in PCS and NR tends to be higher than that prescribed in any risk neutral equilibrium. Risk aversion is one possible explanation for this observation. There is evidence from other private goods auction experiments, and from field data, that subjects may be risk averse. For the same reason as in those cases, risk aversion pushes up equilibrium bids in our hybrid mechanisms.

As a rudimentary look at risk aversion as a possible explanation, we recalculated the equilibrium sets for the three rules if the players have a utility function given by $U_{i}(a)=\left(v_{i} x_{1}-s_{i}\right)^{r}, r=3 / 4,1 / 2$ and $1 / 4$. For SCS, there is no effect at all because truthful reporting is a dominant strategy equilibrium. However, risk aversion has a non-neutral effect for both PCS and NR. Intuitively, the effect of risk aversion should be to increase bids, for exactly the same reason as in private value auctions (Goeree et al., 2002). Increasing one's bid increases the probability of providing the public good, but increases the expected payment, too. At the margin, a risk averse player will pay more than a risk neutral player to

\footnotetext{
15 There are other possible factor that were uncontrolled and could have led to overbidding. These include judgement biases, or misperceptions of conditional probabilities, and different possible dimensions of social utility, ranging from altruism to concerns over relative earnings instead of expected earnings.
} 
Table 9

Proportions of bids above value

\begin{tabular}{llllllll}
\hline PCS & \multicolumn{2}{l}{} & & & NR & \\
\cline { 1 - 2 } Value & 29 & 45 & 90 & & 29 & 45 & 90 \\
\hline Rds. 1-5 & 0.076 & 0.128 & 0.025 & & 0.038 & 0.000 & 0.000 \\
Rds. 6-10 & 0.088 & 0.126 & 0.021 & & 0.000 & 0.000 & 0.000 \\
All rds. & 0.082 & 0.127 & 0.023 & & 0.018 & 0.000 & 0.000 \\
\hline
\end{tabular}

reduce downside risk (i.e. the risk of non-provision), so they are willing to bid higher. Thus, risk aversion creates upward pressure on bids, leading to more frequent provision and more frequent overpayment, relative to the predictions based on risk neutrality. Figs. 6 and 7 give a three dimensional graph of the equilibrium sets for PCS and NR, respectively, for $r=1 / 2$.

As Tables 12a-12c shows, risk aversion increases the upper range of bids that are consistent with equilibrium. While some low bids are still consistent with equilibrium for risk averse bidders, higher bids, which were inconsistent with risk neutral equilibrium behavior, are consistent with risk averse equilibrium behavior. As a consequence, as Tables $12 \mathrm{~b}$ and $12 \mathrm{c}$ indicate, risk aversion is indeed one way to account for most of the bids in excess of the risk neutral Nash equilibrium bids. For example, for square root utility functions in PCS for $v=45,70 \%$ of empirical bids were in the equilibrium range of bids over the first five rounds, $54 \%$ over the second five rounds and $62 \%$ over all rounds.

Risk aversion has a noticeable effect on the empirical proportion of bids falling in the equilibrium range, increasing it by 5-27 percentage points. Except for the values 29 and 45 in PCS, this proportion is quite high for $r=1 / 4$. These two exceptions are due to the relatively high proportion of bids above value in these cases (see Table 9). Of the bids that a rationality-based theory could explain (i.e., bids no greater than value), for $v=29$ the proportion increases from 0.81 under risk neutrality to 0.86 under $r=1 / 4$. For $v=45$, it increases from 0.61 under risk neutrality to 0.87 under $r=1 / 4$.

Thus, the results are suggestive risk aversion is a good explanation for the higherthan-expected bidding behavior, but not conclusive. Risk aversion is only one of several possible explanations, and other modification so the basic model, such as allowing for other-regarding preferences (altruism or equity), are interesting alternatives.

\subsubsection{Learning}

Another family of possible explanations for overbidding is that it emerges as part of a dynamic process of convergence to equilibrium. One learning hypothesis is that

Table 10

Empirical median bid functions

\begin{tabular}{llllllll}
\hline PCS & & & & NR & \\
\cline { 6 - 8 } Value & 29 & 45 & 90 & & 29 & 45 & 90 \\
\hline Rds. 1-5 & 22 & 35 & 55 & 21 & 30 & 50 \\
Rds. 6-10 & 23 & 37 & 55 & 20 & 30 & 48 \\
All rds. & 22 & 35 & 55 & & 20 & 30 & 49 \\
\hline
\end{tabular}


Table 11

Zero bids as proportion of all bids

\begin{tabular}{llllllll}
\hline PCS & & & & \multicolumn{2}{l}{ NR } & \\
\cline { 1 - 3 } Value & 29 & 45 & 90 & & 29 & 45 & 90 \\
\hline Rds. 1-5 & 0.038 & 0.000 & 0.000 & & 0.038 & 0.000 & 0.000 \\
Rds. 6-10 & 0.049 & 0.009 & 0.000 & & 0.000 & 0.000 & 0.000 \\
All rds. & 0.043 & 0.004 & 0.000 & & 0.018 & 0.000 & 0.000 \\
\hline
\end{tabular}

subjects initially begin the experiment bidding too high, due to their misjudgement of probabilities or decision biases, and then adjust their bids downward in the direction of equilibrium, as they gain experience. We tested for trends in behavior in a very rudimentary way, by regressing individual bids on round for each of the three mechanisms, controlling for valuation. If there are no learning effects within a

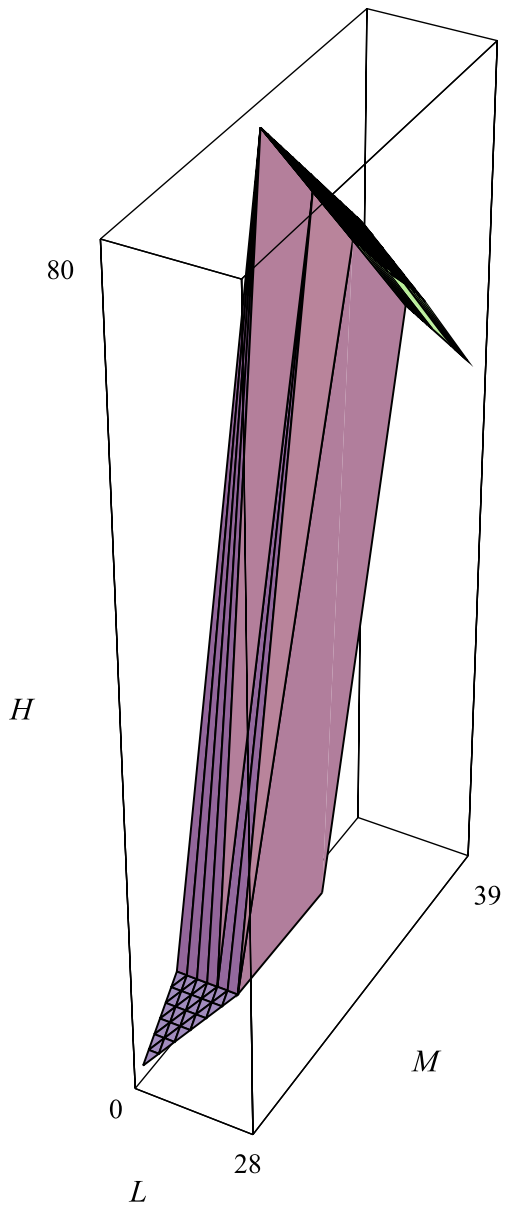

Fig. 6. Equilibrium set, proportional cost sharing mechanism, square root utility. 


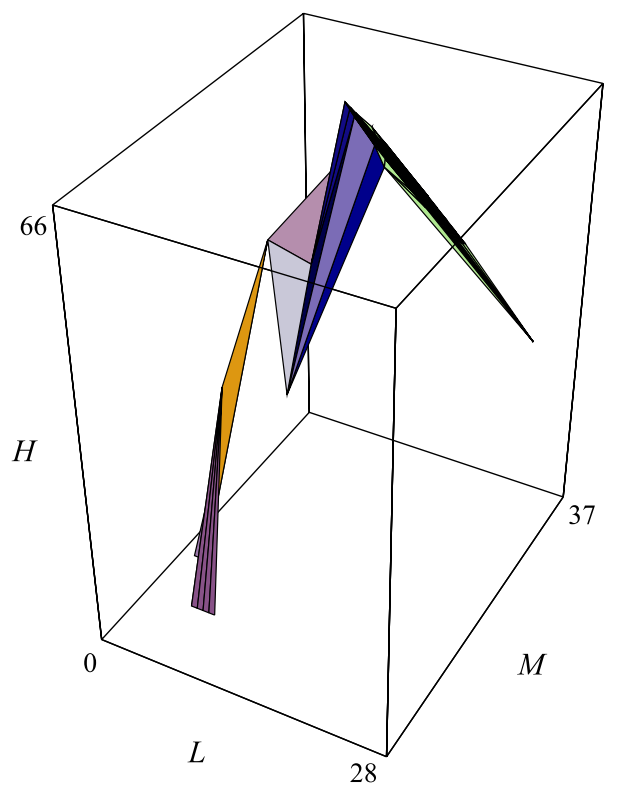

Fig. 7. Equilibrium set, no rebates mechanism, square root utility.

mechanism, each of these six lines plotting the bid as a function of round should have slope $=0$. In all six cases, OLS regressions cannot reject this null hypothesis at the 5\% level and rejection occurs for only one in six at the $10 \%$ level. The coefficients and standard errors are listed in Table 13. From Table 13, one can see that the consistency of bidding behavior with Nash equilibrium increases slightly between the early rounds and the later rounds, but this change is small in magnitude, except for the lowest valuation bidders. There is also little evidence of learning in our SCS data, using a similar approach. Therefore, while subjects may be adapting slightly as they gain experience, learning effects on bidding behavior are not manifested in a strong trend toward lower bids over time.

However, there is some evidence of a different kind of learning effect in our data, which is a sequencing effect. Recall that two mechanisms were run, in sequence, in each session.

Table $12 \mathrm{a}$

Equilibrium bid range by selected utility function exponents

\begin{tabular}{llllllll}
\hline PCS & \multicolumn{2}{l}{} & & & NR \\
\cline { 7 - 8 } Value & 29 & 45 & 90 & & 29 & 45 & 90 \\
\hline Risk neutral & $0-26$ & $0-36$ & $40-62$ & & $0-26$ & $0-35$ & $34-54$ \\
$R=3 / 4$ & $0-27$ & $0-37$ & $46-72$ & & $0-28$ & $0-37$ & $37-58$ \\
$R=1 / 2$ & $0-28$ & $0-39$ & $46-80$ & & $0-28$ & $0-37$ & $46-66$ \\
$R=1 / 4$ & $0-28$ & $0-40$ & $46-88$ & & $0-28$ & $0-40$ & $46-76$ \\
\hline
\end{tabular}


Table $12 b$

Empirical proportion of bids in equilibrium bid range, PCS

\begin{tabular}{llll}
\hline PCS & & & \\
\hline Value & 29 & 45 & 90 \\
\hline Risk neutral & $0.74,0.75,0.74$ & $0.59,0.49,0.54$ & $0.63,0.68,0.65$ \\
$R=3 / 4$ & $0.76,0.76,0.76$ & $0.63,0.52,0.57$ & $0.50,0.56,0.53$ \\
$R=1 / 2$ & $0.78,0.79,0.79$ & $0.70,0.54,0.62$ & $0.57,0.62,0.59$ \\
$R=1 / 4$ & $0.78,0.79,0.79$ & $0.77,0.74,0.76$ & $0.59,0.63,0.60$ \\
\hline
\end{tabular}

Rds. $1-5$, rds. 6-10, all rds.

Behavior in PCS, for $v=29$ and $v=45$, is mildly sensitive to which mechanism was run first in the experimental sessions. The sequence effect on NR appears weaker. Bids in PCS are less variable when it is run second than when it is run first. For $v=45$, the bid CDF for PCS second is nearly first order stochastically dominated by that for PCS first. Also, there is no bidding above value in PCS when it was run second, while it is occasionally observed in sessions when it is run first. This is consistent with a more general finding that ovebidding declines with experience. For NR, generalization seems more difficult. For $v=29$, the bid CDF for NR second is first order stochastically dominated by that for NR first. For $v=45$ in NR, there is no clear effect.

The SCS mechanism was never run as the second treatment within a session, which might cause concern because of the asymmetry of the design. However, there are at least two reasons why this is not a problem in our experiment. First, since players have dominant strategies, behavior in SCS was very close to the predicted equilibrium, and if anything one would expect behavior to be even closer to equilibrium if SCS were run as a second treatment. This was one of the main reasons we chose to run SCS first. It is the simplest mechanism for the subjects, in terms of cognitive difficult and strategic thinking. Second, if anything, our conclusions are even stronger because of this design feature. As we show in the next session, SCS had lower efficiency than the other mechanisms. Since SCS was always run first, and since subjects generally tend to overbid before they were experienced, the net effect would be to increase the efficiency of allocations under SCS. In fact, this appears to have happened in our experiment. This is confirmed by Tables 6 and $7 .^{16}$

\subsection{Groups: efficiency comparisons}

A central reason for conducting these experiments was to compare these mechanisms on efficiency grounds. The SCS mechanism has been defended on two grounds. First, it has very attractive incentive properties: it is strategy proof. Second, it is more efficient than any other strategy proof, individually rational mechanism! However, this leaves open the question of how efficiently it performs in comparison to other simple mechanisms with less attractive incentive properties (i.e. which are not strategy proof). The PCS and NR mechanisms are indeed very simple and shares with SCS the attractive feature of individual rationality. Moreover, from the revelation principle, we know that

\footnotetext{
${ }^{16}$ Furthermore, the subjects actually earned more in the SCS treatment that they would have if everyone had played a dominant strategy.
} 
Table 12c

Empirical proportion of bids in equilibrium bid range, NR

\begin{tabular}{llll}
\hline NR & & & \\
\hline Value & 29 & 45 & 90 \\
\hline Risk neutral & $0.78,0.86,0.82$ & $0.85,0.85,0.85$ & $0.64,0.67,0.65$ \\
$R=3 / 4$ & $0.85,0.95,0.90$ & $0.89,0.89,0.89$ & $0.64,0.67,0.65$ \\
$R=1 / 2$ & $0.85,0.95,0.90$ & $0.89,0.89,0.89$ & $0.47,0.43,0.45$ \\
$R=1 / 4$ & $0.85,0.95,0.90$ & $0.98,10.00,0.99$ & $0.70,0.49,0.60$ \\
\hline
\end{tabular}

Rds. 1-5, rds. 6-10, all rds.

the equilibria of PCS and NR correspond to direct mechanisms that are Bayesian incentive compatible (although not strategy proof). Theoretically, there exist equilibria of each of these mechanisms that are more efficient than the outcomes generated by serial cost sharing. Of course, there is a severe multiple equilibrium problem in these mechanisms, which may lead to uncertainty and doubt about performance. In this section, we compare the efficiency of the outcomes in our experiment, across the three mechanisms.

There are several ways in which efficiency comparisons can be made. We focus on two: (a) the proportion of available total surplus extracted by each mechanism and (b) the proportion of times each mechanism makes the efficient decision. Both comparisons lead to the same conclusion. PCS significantly outperforms SCS and NR, with the latter two leading to approximately the same efficiency levels.

\subsubsection{Surplus extraction}

With total surplus extraction as the criterion, the PCS mechanism was clearly the best performing of the three mechanisms. As Table 14 shows, for almost all value profiles, PCS performs at least as well as and sometimes much better (up to 50 percentage points) than SCS. The main exception is the profile $\langle 45,45,45\rangle$, which is the only one where SCS outperforms PCS, and this difference is based on a very small number of observations. Interestingly, SCS and PCS surplus extractions move in similar ways as the type profile varies, but SCS changes much more sharply with profiles. This gives it a "hit or miss" character: it typically either does very well at extracting surplus or it does very poorly. This is due to the relatively low variance in the underlying bids. PCS outperforms NR, for all profiles, a difference that is attributable to the consistently higher bids under PCS than NR. The SCS and NR mechanisms perform almost

Table 13

Learning regressions $\left(*\right.$ significant $^{\mathrm{a}}$ at $\alpha=0.10$ )

\begin{tabular}{lll}
\hline Value & PCS $($ OLS $)$ & NR $($ OLS $)$ \\
\hline 29 & $0.000(0.311)$ & $-0.481^{*}(0.254)$ \\
45 & $-0.257(0.301)$ & $0.125(0.257)$ \\
90 & $-0.329(0.401)$ & $-0.252(0.408)$ \\
& $n=660$ & $n=360$ \\
\hline
\end{tabular}

\footnotetext{
${ }^{a}$ Statistical significance is probably overstated because the observations are not truly independent.
} 
Table 14

Total surplus extraction at each profile, by mechanism

\begin{tabular}{llllr}
\hline Surplus extraction & \multicolumn{1}{l}{} & \\
\hline Profile & PCS & SCS & NR & Available surplus \\
\hline$\langle 29,29,45\rangle$ & $0.067(30)$ & $-2.30(12)$ & $0.083(12)$ & 1 \\
$\langle 29,45,45\rangle$ & $0.379(29)$ & $0.111(9)$ & $0.00(16)$ & 17 \\
$\langle 45,45,45\rangle$ & $0.692(13)$ & $1.00(6)$ & $0.00(7)$ & 33 \\
$\langle 29,29,90\rangle$ & $0.500(9)$ & $0.00(11)$ & $0.111(9)$ & 46 \\
$\langle 29,45,90\rangle$ & $0.595(42)$ & $0.191(22)$ & $0.400(25)$ & 62 \\
$\langle 45,45,90\rangle$ & $0.905(22)$ & $1.00(8)$ & $0.857(7)$ & 78 \\
$\langle 29,90,90\rangle$ & $0.917(24)$ & $0.729(11)$ & $0.813(16)$ & 107 \\
$\langle 45,90,90\rangle$ & $0.968(32)$ & $1.00(12)$ & $0.889(18)$ & 123 \\
$\langle 90,90,90\rangle$ & $1.00(11)$ & $1.00(7)$ & $1.00(6)$ & 168 \\
Overall & 0.836 & 0.684 & 0.691 &
\end{tabular}

Entries cells of columns 2-4 are proportions of possible surplus extracted.

Column 6 lists total available surplus for each profile. Number of observations in parenthesis.

identically, although there is some minor profile-specific differences. These conclusions are also supported by a comparison of surplus over all profiles, given in the last row of Table 14.

Clearly, there are differences across the three mechanisms. In order to test for significance of these differences, we used a Tobit model, since the data is censored, with extraction rates constrained to be less than or equal to 1 . The unit of observation was one group of a round in a session, so we have a total of 426 observations. The Tobit results are presented in Table 15. We estimated two models, with slightly different dependent variables. In model 1 , the dependent variable is simply the proportion of possible consumer surplus that is extracted. ${ }^{17}$ Since the actual type-profile varies across groups and production is actually inefficient for some profiles, we control for this variation by including a variable called "profile" that equals the sum of values that are drawn for the group. The Tobit results (Table 15, model 1; constant is SCS) support our conclusions, as well as Hypotheses 4a and 6a (PCS vs. SCS and PCS vs. NR, respectively). Hypothesis 5a (NR vs. SCS), however, is rejected. Relative to SCS, NR had a significant negative effect on consumer surplus holding the profile constant, while PCS had a significant positive effect. In model 2 , the dependent variable is the proportion of possible total surplus that is extracted. The results of model 2 differ in one important respect from the results of model 1. The NR coefficient is now significantly positive instead of significantly negative. This indicates that it is lack of budget balance that results in lower consumer surplus for NR, rather than problems of incentive compatibility. That is, consumer surplus is lower in NR than with SCS only because the consumer surplus measure assumes that overpayments are completely deadweight loss. If the overpayment is viewed simply as a transfer rather than deadweight loss, then we find that NR is actually more efficient than SCS. ${ }^{18}$

\footnotetext{
17 Observations where possible consumer surplus is negative are excluded.

18 The statistical significance of these conclusions relies on an assumption that observations are independent. Because the observations in our dataset have some dependence, the standard errors are underestimated somewhat. However, the significance levels on most variables are small enough that dependencies in the data are unlikely to affect the findings.
} 
Table 15

Tobit results, efficiency comparisons

\begin{tabular}{lll}
\hline & Consumer surplus (model 1) & Total surplus (model 2) \\
\hline Profile & $0.071^{* * *}(0.008)$ & $0.11^{* * *}(0.011)$ \\
NR & $-0.66^{* *}(0.29)$ & $0.54 *(0.34)$ \\
PCS & $1.28^{* * *}(0.28)$ & $1.48^{* * *}(0.32)$ \\
Constant & $-3.04^{*}(0.47)$ & $-4.98^{* * *}(0.61)$ \\
& $n=426$ & $n=426$ \\
\hline
\end{tabular}

Entries are coefficients, standard errors in parentheses.

Significance levels: ${ }^{*} p=0.10, * * p=0.05, * * * p=0.001$.

\subsubsection{Proportion of efficient decisions}

A somewhat coarser view of efficiency is the proportion of successful decisions under each mechanism. For each group-round, we recorded a 1 if the group made the efficient production decision and 0 otherwise. For SCS, exclusion of the $v=29$ member in a $\langle 90,90,29\rangle$ profile did not result in a 0 , to make it harder for other mechanisms to outperform it. Otherwise, the results mirror the findings of Table 14. The results are presented in Table 16.

This table reinforces the conclusions based on surplus extraction. PCS outperforms SCS (supporting Hypothesis 4b), which outperforms NR (rejecting Hypothesis 5b, supporting Hypothesis 6b). However, this "hit rate" analysis does not capture the fine details of mechanism performance. For example, as noted earlier, most of the difference between NR and SCS hit rates is due to the $\langle 29,29,45\rangle$ profile where a hit generates almost zero surplus.

\subsubsection{Robustness of efficiency results to other environments}

These efficiency results are for the specific parameters we chose for our experiment, which leaves open the question of robustness across arbitrary environments. Recall that we chose our distribution of valuations so that the amount of surplus varied a lot across different profiles. Thus, there is a lot of uncertainty in our environment. It is possible that in an environment with less uncertainty, for example if the likelihood of a 45 valuation was very high, SCS may have performed better because it was more successful at $\langle 45,45,45\rangle$ than the auction mechanisms. However, at profiles other than this one, the relative ranking of PCS and SCS on efficiency grounds was very consistent, suggesting that the ranking we find is fairly robust. Compared to NR, SCS does well in high-surplus profiles, while NR does better in low-surplus profiles. This suggests that in environments with sufficient heterogeneity of valuations, auction mechanisms, such as PCS, could be expected to perform better than SCS.

Another aspect of the environment is the number of agents in the model. Our experiment considered a small group and leaves open the question of performance in larger groups. The incentive problems of PCS will become worse as group size increases. The exclusion inefficiencies also become worse for SCS, so the question is which effect dominates. We conjecture that the group size effect will tend to favor SCS over PCS, so that for sufficiently large groups, PCS will not perform as well as SCS. However, a version of PCS that allows for exclusion will do better than SCS. 
Table 16

Average success rate, by mechanism (standard errors in parenthesis)

\begin{tabular}{lll}
\hline PCS & NR & SCS \\
\hline $0.650(0.032)$ & $0.475(0.046)$ & $0.520(0.050)$ \\
\hline
\end{tabular}

An example would be an auction mechanism where bidders are excluded if their bids fall below a reserve bid. In the limit, as the number of bidders goes to infinity, incentive compatibility will eventually imply equal cost shares (e.g. Ledyard and Palfrey, 1999a,b, 2002), so that optimal auction mechanisms for public goods will look similar to SCS.

\section{Conclusions}

This paper reports the results from a laboratory experiment designed to compare the performance of the serial cost sharing mechanism to two public good auction mechanisms that are not incentive compatible. These alternative mechanisms are both simple to understand and to implement, they are individually rational, and one of them is budget balancing. We fully characterize the set of Bayesian Nash equilibria under the auction mechanisms, for our experimental environment, and establish some general properties of the equilibrium sets. The three mechanisms have systematic theoretical differences both in terms of the individual and collective behavior they induce. These theoretical properties are reflected in the data.

The dominant strategy equilibrium in SCS explained subject behavior fairly well. Deviations from dominant strategy can be accounted for by quantal response equilibrium. PCS induced significantly higher bidding than NR and bidding above value was quite rare in NR. The relatively high bidding behavior parallels a well-documented phenomena in private good auctions (Goeree et al., 2002). Risk aversion is one possible explanation for overbidding. All these findings, with the exception of systematic overbidding, supported our main hypotheses. There was evidence of learning, with the one exception that bids in PCS are lower among subjects who have previously participated in the NR mechanism.

As hypothesized, SCS was outperformed on welfare grounds-both the surplus extracted and the likelihood of an efficient decision - by PCS, in spite of the fact that PCS is not incentive compatible and is plagued with multiple equilibria. We find this result interesting, because of the combination of incentive problems and coordination problems in PCS, and it suggests that more research on auction-like public goods mechanisms is warranted. The key advantage of PCS is that it never inefficiently excludes any users of the public good, and it allows unequal cost shares, so high value users can subsidize low value ones. Thus, it overcomes the primary two sources of inefficiency in SCS applied to public goods. However, it also suffers the disadvantage of having multiple equilibria, some of which could have produced outcomes much worse than SCS. As the experiments demonstrate, the PCS equilibrates close to the better, high-bidding, equilibrium and coordination failure (i.e., convergence to low-bidding equilibria) is avoided. This finding opens interesting theoretical questions about equilibrium selection in these mechanisms. 
Another auction mechanism, NR, which is neither strategy proof nor budget balanced, also overcomes these difficulties, but did not perform as well. The NR mechanism produced efficiency levels about the same as SCS, unless the surplus bids are considered deadweight loss. This indicates that the fine details of the auction mechanism can have important consequences. These findings echo similar results about the role of rebates for nonexcludable public goods, reported in Ferejohn et al. (1982).

These results suggest several directions for further work, both experimentally and theoretically. Experimentally, it would be instructive to compare the performance of PCS and SCS in other environments and to other mechanisms that have interesting theoretical properties. One important example is the pivot mechanism. The standard pivot mechanism is not individually rational and does not balance the budget, but hybrid mechanisms that retain some features of the pivot mechanism, while allowing for exclusion or an opt-out stage, might be interesting to study. Auction procedures that allow for exclusion also warrant consideration. Such mechanisms could offer probabilities of public good consumption; agents would be excluded probabilistically. This could make each agent pivotal more often and enhance efficiency as a result. A plurality voting scheme, in which agents vote over quantities and share $1 / n$ of the cost of the plurality winner, is another interesting and practically relevant procedure.

Finally, the evidence we find indicating the possibility of risk aversion or altruism suggests that the Bayesian mechanism design approach to public good provision should be extended to allow for environments with preferences other than the risk neutral, selfish preferences that are often assumed. One would conjecture that the optimal mechanisms may differ significantly in small groups. This contrasts with the case of very large groups, where the convergence results in Ledyard and Palfrey (2002) indicate that risk aversion will not affect the form of the optimal mechanism.

\section{Acknowledgements}

We thank the National Science Foundation and the Social Science Experimental Laboratory at Caltech for financial support. We are grateful for helpful comments from Ray Battalio, Matthew Jackson, Richard McKelvey, seminar participants at Texas A\&M University, Carnegie Mellon University and the University of Chicago, participants at the Fall 2000 meetings of the Economic Science Association and two anonymous referees. Thanks also to Serena Guarnaschelli, Elena Asparouhova and Vale Murthy for help running the experiments, and to Julie Malmquist and the Social Science Department at Pasadena City College for their help in recruiting subjects for the experiment.

\section{Appendix A. Instructions}

\section{A.1. Decision making experiment}

This is an experiment in group decision making. You will be paid for your participation in cash, at the end of the experiment. Your payment depends partly on your decisions, 
partly on the decisions of others and partly on chance. No other participant will be told how much you earn in the experiment, and you are not obligated to tell anyone else how much you earn. To keep track of your earnings during the experiment, we use an experimental currency, which we call francs. Francs are then redeemed for cash at the end of the experiment. Each franc is worth $E R$ dollars.

We will start with a brief instruction period, during which the experiment, the decisions you are to make and how you earn money will be explained to you. Please listen carefully and raise your hand if you have a question. DO NOT write anything down yet and do not pick up the die. After the instructions, we will have two practice rounds to familiarize you with the decision making procedure.

All interaction between you will take place via your decision sheets. Please do not try to communicate with each other in any other way; if you do, you will be asked to leave the experiment and will not be paid.

We will now pass out your decision and record sheets. The first of these sheets is for the first part of the experiment, on which you will record your decisions and payoffs for each round. We will collect these after the first part of the experiment is over. The second is a record sheet for the whole experiment, which you will use keep track of your earnings throughout the entire experiment.

We will also assign subject numbers, starting in the front on your right with $\# 1$, to help keep track of which decision sheet belongs to which participant. Please write your subject number in the space provided on both of your record sheets.

\section{A.2. Serial cost sharing}

The experiment consists of a sequence of rounds and, in each round, you will be anonymously grouped with two other participants. To determine the groupings in the first round, numbered ping pong balls will be drawn out of a box, three at a time. The subjects with the first three numbers drawn will in the group 1, subjects with the next three numbers drawn will be in group 2 and so forth. You will NOT see the numbers on the balls that are drawn; therefore, you will not know who is in your group. Thus, decisions in the experiment are anonymous in the sense that no other participant will know what group you are in or what decisions you make.

In the second round and in each subsequent round, we will again draw the ping pong balls three at a time to determine new group assignments. Therefore, in each round, each of you are assigned to exactly one group. What happens in the groups you do not belong to has no effect on your payoff. In this sense, groups are completely independent of each other.

In each round, your group of three participants must decide whether or not to purchase a fictitious good called a gadget, and exactly which members of your group are allowed to use the gadget. For your group to purchase a gadget, your group must pay a total of 102 francs. It is possible that not everyone in your group is allowed to use the gadget. Those members of your group who are allowed to use the gadget are called users and pay an equal share of the cost. Those members of your group who are not allowed to use the gadget are called nonusers and do not pay any share of the cost. Therefore, if all members are users, the cost is split three ways, so each member pays 34 francs. If only two members 
are users, the cost is split two ways, and each of the two users pays 51 francs, and if only one member is a user, then this user pays the entire 102 francs.

If you are a user in a round, you earn for that round an amount called YOUR USER VALUE. Your user value is determined at the beginning of each round in the following way. At the beginning of each round, we will come to you privately one at a time and let you throw a fair six-sided die. If you roll a 1 or 2 , your user value will be 29 francs. If you roll a 3 or 4 , your user value will be 45 francs. If you roll a 5 or 6 , your user value will be 90 francs. [write on board] Your user value determines how much you earn in francs if your group buys the gadget and you are a user. Record this user value in the space of your record sheet labeled YOUR USER VALUE, on the line corresponding to the round. Notice that no one else in your group knows your user value and you do not know the user value of anyone else in your group. You only know your own user value. If you are a non-user in a round, you earn zero in that round, regardless of what your user value is.

To determine whether your group buys the gadget in a round, every member of the group will simultaneously submit a message. Every participant must choose one of three messages: high, medium or low. You are free to submit any of these three messages in any round. To submit your message, record it on your record sheet in the column labeled YOUR MESSAGE and we will collect all the sheets.

The messages are used to determine whether your group buys the gadget and which members are users. If you submit "low" then you are a non-user and your earnings for that round are zero, regardless of the messages other members send. If you send "middle" that means that you are willing to be a user only if the cost is split three ways. "High" means that you are willing to be a user if the cost is split either two or three ways. The table on the board summarizes how messages are converted into purchase decisions.

Thus, if you announce low in a round, you will not have to pay anything, but you will not earn anything even if your group purchases the gadget. If you announce medium, you will pay 34 francs of the cost - a three-way split - and receive your user value, only if the group purchases the gadget. This purchase will occur as long as the other two member of your group both announce either medium or high. Similarly, if you announce high and no member announces low, you will pay 34 francs - a three-way split - and receive your user value. However, if you announce high and exactly one other member announces high and one other member announces low, your group will purchase the gadget. In this one case, you will pay 51 francs - a two-way split - and receive your user value. This information is summarized in the table on the board.

To help you understand this table, we will now go through each line of it. Every possible set of three messages is depicted on the table. Therefore, the table shows every possible combination of group decisions, number of users, and the share of the cost users must pay. The first line shows the outcome if all three messages in a group are HIGH. In that case, everyone claims to be willing to share up to half the cost of the gadget; therefore, all three members have an equal one-third share of the cost, 34 francs. The second line shows the case where the messages are two HIGH and one MEDIUM. The high messages imply a willingness to share at least one-third of the cost and up to one-half if necessary; the medium messages implies a willingness to share no more than one-third of the cost. Therefore, all three members are users and have an equal share of the cost, 34 francs. If the messages are as in the third and fourth lines - one HIGH and two MEDIUM, or three 
MEDIUM - again all group members claim to be willing to share no less than one-third the cost of the good. Again, this means that all three members are users and all have an equal 34 francs share of the cost. In the fifth line, there are two HIGH messages and one LOW. Two members are willing to share up to one-half the cost of the gadget, while one member is not willing to share any of the cost. This member is therefore not a user and does not share any of the cost. The two members who send HIGH messages, however, are users, and they will split the cost equally between the two of them- 51 francs each. These five lines reflect ever possible way the group can purchase the good. In the next line, there is one message of each type, high, medium and low. Therefore, one member is willing to share up to one-half the cost if necessary, one member is willing to share one-third the cost but no more, and one member is willing to share none of the cost. This is not enough to cover the whole cost, so the group does not purchase the gadget. Therefore, no one is a user and there is no cost to share. The next line is similar: one member is willing to share up to one-half the cost, but the other two claim to be willing to share none of it. This is not enough to cover the whole cost, so the group does not purchase, no one is a user, and there is no cost to share. In the next three lines, no member is willing to share up to one-half the cost. In line 7, two are willing to share one-third and one is willing to share none. In line 8, one is willing to share one-third and two are willing to share none. In both cases, there is not enough to cover the cost, so the group does not purchase the gadget, no one is a user, and there is no cost to share. Finally, in line 9, no member is willing to share any of the cost - therefore, the group does not purchase the gadget, no one is a user, and there is again no cost to share. These five lines exhaust all the possible ways a group can decide not to purchase the gadget.

Your decision and record sheet is now displayed on the overhead. The first column lists the round. The next column is where you will record YOUR USER VALUE for a round, which you will determine by the throw of a die at the start of each round. After you learn your value, you will decide on a message, and in each round write it in the third column. We will then collect the decision sheets and fill in for you the messages of your other group members, which will be listed in the next two columns. The decisions sheets will then be returned, so that YOU can calculate your cost share, whether you are a user and your payoff. When this is complete we will go on to the next round.

I will now explain how to calculate whether your are a user and your cost share in any round. Please listen carefully, as if you record your cost share incorrectly, you may shortchange yourself. In every round, to calculate your cost share, find the one of the following cases that applies to you and record the information on your record sheet:

1. If YOUR MESSAGE is LOW, your cost share is ZERO and you are not a user. This is true no matter what the other messages are in your group.

2. If YOUR MESSAGE is MEDIUM and the neither of the other two messages in your group is LOW, then you are a user. Your cost share is 34 .

3. If YOUR MESSAGE is MEDIUM and at least one of the other two messages in your group is LOW, you are not a user. Your cost share is ZERO.

4. If YOUR MESSAGE is HIGH and neither of the other two messages is low, you are a user. Your cost share is 34 . 
5. If YOUR MESSAGE is HIGH and one of the other two messages is LOW and the other is HIGH, you are a user. Your cost share is 51.

6. If YOUR MESSAGE is HIGH and at least one of the other messages is LOW, and neither of the other messages is HIGH, then you are not a user. Your cost share is ZERO.

In each round, you will fall into exactly one of these cases. We will leave this up for the duration of the experiment so you can determine your cost share easily.

We will now go through two practice matches, to help make the procedures more concrete. Please note that you are not paid for the practice matches. When you have written down your decision in each round, place your decision sheet UPSIDE DOWN on top of your monitor so we can come pick it up.

We will now proceed to practice round $\mathrm{P} 2$. This practice round is exactly like practice round P1. New groups will be formed using the ping pong balls, each of your new values will be randomly assigned by rolling a die in private, and you will be asked to decide which message to send.

We will now proceed to the first actual round. There will be 10 rounds. Each round will be conducted exactly like the two practice rounds.

The first part of the experiment is now over. Please add your payoffs in francs from each round and write the total in the space on the Part I record sheet. Then multiply this total in francs by the exchange rate to get your dollar earnings from Part I. Write down your dollar earnings in the space provided both on your Part I record sheet and the whole experiment record sheet. We will then collect the Part I record sheets and distribute record sheets for Part II.

\section{A.3. Proportional cost sharing}

In this part of the experiment, the determination of your group and your user value are the same as before, but the decision making and cost sharing procedures are different.

As before, you will be assigned a new value at the beginning of each round by rolling a die. The cost for your group to purchase the gadget is still 102 francs. Now, however, instead of having only three messages, high, medium or low, your message will be an integer from 0 to 102 , which we will call YOUR BID. In any round, you may submit any integer you like in this range. If the sum of the bids in your group is at least 102, your group purchases the gadget and everyone is a user, regardless of what bid they submit. If the sum of bids in your group is less than the cost of the gadget, your group does not purchase the gadget.

If your group purchases the gadget in a round, you will be paid your user value for that round. However, you will also have to pay a share of the cost. Specifically, your share of the cost will be the ratio of your bid to the sum of all three bids in your group, times 102 francs. In other words, you will share a fraction of the 102 francs cost that is proportional to your bid. If the sum of the bids is 102, you pay your bid. If the sum is greater than 102, you pay less than your bid. Note, therefore, that you will never have to pay more than your bid. 
If your group does not purchase the gadget in a round, there is no cost to share and you do not get your USER VALUE of the gadget; therefore you are paid 0 franc.

Therefore, your payment in every round will be:

- 0 franc if your group does not purchase the gadget, i.e., (YOUR BID+OTHER 2 BIDS) $<102$

- YOUR VALUE-102*(YOUR BID)/(YOUR BID+OTHER 2 BIDS) if your group does purchase the gadget, i.e., if (YOUR BID+OTHER 2 BIDS) $\geq 102$

To sum up, there are three specific differences from the first part:

1. Your bid is any number between 0 and 102, rather than just a message high, medium or low.

2. Different people in the group may have different shares of the cost - the cost is not automatically split equally among users.

3. There is no difference between users and non-users. If your group purchases the gadget, you automatically use it, and therefore you get your value for the gadget, no matter what your bid was for that round.

Your decision and record sheet for Part II is displayed on the overhead. PLEASE WRITE YOUR SUBJECT NUMBER IN THE SPACE AT THE TOP. Again, in each round, you will record your value in the second column, and then your bid in the third. When all bids have been recorded, we will collect the decision sheets and fill in the columns with the bids of your group members, as well as the sum of ALL THREE BIDS in your group, including yours. Decision sheets will then be returned so that YOU can calculate your cost share and your payoff. Note that you will not calculate whether you are a user or not, because if the sum of the bids is greater than 102, you are automatically a user. In making all calculations, round to the nearest whole number.

To help you calculate your cost share in any round, always follow the following steps. It is important that you listen carefully so that you are paid what you truly earn in the experiment.

1. If the sum of the bids is LESS THAN 102, your cost share is ZERO and you do not use the gadget.

2. If the sum of the bids is AT LEAST 102, then to calculate your cost share you:
A. enter YOUR BID in your calculator
B. press $*$ or $\times$ on the calculator
C. press 102
D. press $=$
E. press $/$ or $\div$ on your calculator
F. enter the sum of bids in your group
G. $\quad$ press $=$ 
The number that appears next is your cost share. If this cost share is not a whole number, round it to the nearest whole number. Write the result on your record sheet. In other words: to find your cost share, multiply your bid by 102 and divide the result by the sum of bids in your group. If your answer is ever greater than your bid, you made a mistake. To make this part more concrete, we will have two practice matches. The practice matches do not count toward your earnings.

\section{A.4. No rebates}

In this part of the experiment, everything is as before except the rule for calculating your cost. The determination of your group and your user value are the same as before, as are the bids you can make and the rule to determine whether you are a user. Only the cost sharing procedure is different.

If the sum of bids is at least 102 so your group does purchase the gadget, your cost is simply YOUR BID. In every round where your group purchases the gadget, you are paid your USER VALUE for that round minus YOUR BID for that round. NOTE that, if your bid in any round is larger than your user value for that round AND your group purchases the gadget, you will LOSE MONEY in that round. If your group does not purchase the gadget in a round, that is, the sum of bids in your group in that round is less than 102, there is no cost to share and you do not get your USER VALUE of the gadget; therefore, you are paid 0 franc.

In other words: if the sum of the bids in your group is at least 102, you are paid your user value AND you will have to pay your bid. This is true regardless of how much you bid. If the sum of bids in your group is less than 102, you are paid 0 and you do not have to pay anything. Thus, the bids are used to determine both whether your group purchases the gadget and what your cost will be.

Therefore, your payment in every round will be:

- 0 franc if your group does not purchase the gadget, i.e., (YOUR BID+OTHER 2 BIDS) $<102$

- YOUR VALUE-YOUR BID if your group does purchase the gadget, i.e., if (YOUR BID+OTHER 2 BIDS) $\geq 102$

Your decision and record sheet is now displayed on the overhead and I will now explain how to fill it out. The first column lists the round of the experiment. The next column is where you will record YOUR USER VALUE for a round, which you will determine by the throw of a die at the start of each round. After you learn your value, you will decide on a bid, and in each round write it in the third column. When all bids have been recorded, we will collect the decision sheets and fill in the columns with the bids of your group members, as well as the sum of ALL THREE BIDS in your group, including yours. Decision sheets will then be returned so that YOU can calculate your cost share and your payoff. When you have calculated your cost share and payoff, write them in the spaces provided for that round. In making all calculations, round to the nearest whole number.

We will now go through two practice matches, to help make the procedures more concrete. Please note that you are not paid for the practice matches. When you have 
written down your decision in each round, place your decision sheet UPSIDE DOWN on top of your monitor so we can come pick it up.

We will now proceed to practice round $\mathrm{P} 2$. This practice round is exactly like practice round P1. New groups will be formed using the ping pong balls, each of your new values will be randomly assigned by rolling a die in private and you will be asked to decide which message to send.

We will now proceed to the first actual round. There will be 10 rounds. Each round will be conducted exactly like the two practice rounds.

\section{Appendix B. Proofs of Propositions 6-8}

Proof of Proposition 6. In PCS, $P\left(b^{L}\right), P\left(b^{M}\right)$ and $P\left(b^{H}\right)$ are strictly positive in any nontrivial symmetric equilibrium.

Proof. Focus on $P\left(b^{M}\right)$, as $P\left(b^{H}\right)$ must be strictly positive if $P\left(b^{M}\right)$ is. If $P\left(b^{M}\right)=0$, then only at the profile $\langle 90,90,90\rangle$ is the public good produced (or else the equilibrium is trivial). Then the equilibrium bid $b^{H}$ can only be 34 . But $v=45$ has a profitable deviation to $b^{\prime}=34$ in that case, which contradicts the assumption of equilibrium.

If $P\left(b^{L}\right)=0$ in a nontrivial symmetric equilibrium, then $b^{H}<51$. In any such strategy profile, some type has a profitable deviation. Consider two cases. First, if $b^{H} \in[37,51]$, then $v=29$ has a profitable deviation to $102-2 b^{H}$, contradicting the assumption of equilibrium. Second, if $b^{H}<37$, then $b^{M} \geqslant 30$, since $\langle 45,90,90\rangle$ produces in any equilibrium where $\langle 90,90,90\rangle$ produces. Further, $b^{H} \geqslant 34$ or else $\langle 90,90,90\rangle$ does not produce. But for a bid function $\{l, m, h\}, h \in[34,36], m \in[30, h], l \in[0,102-2 h), v_{i}=90$ has a profitable deviation to $102-m-l$. With this deviation, at $v_{-i}=(90,90),(90,45),(45$, $90)$ and $(45,45), v_{i}=90$ 's payoff goes from 56 to 39 , but at profiles $v_{-i}=(45,29),(29,45)$, $(90,29)$ and $(29,90), v_{i}=90$ 's payoff goes from 0 to 22 . This contradicts the assumption of equilibrium.

Proof of Proposition 7. In NR, $P\left(b^{M}\right)$ and $\mathrm{P}\left(b^{H}\right)$ are strictly positive in any nontrivial symmetric equilibrium.

Proof. The proof is the first paragraph of that for the PCS analogue. Unlike for PCS, $P\left(b^{L}\right)=0$ is possible in a nontrivial symmetric equilibrium in NR: $\{0,34,34\}$ is an example.

Proof of Proposition 8. Consider a symmetric profile of bid functions $b=\left\{b^{L}, b^{M}, b^{H}\right\}$ in PCS or NR. If player $i$ with value $v_{i}$ has any interim profitable deviation from $b^{v}$, then one of the following deviations is profitable: $d=0$ or $d=102-x-y$ for some $x, y \in b$.

Proof. We prove this by recursively considering all possible deviations. The order of the recursion is determined by $2 b^{M} \lessgtr b^{L}+b^{H}$.

First consider the case where $2 b^{M}>b^{L}+b^{H}$. Focus on player $i$. Consider a deviation from $b^{v}$ to $d_{1} \in\left[0, \max \left[102-2 b^{H}, 0\right]\right]$. All bids in this interval result in the same 
production decision as $d_{1}^{\prime}=0$, and $d_{1}^{\prime}=0$ never costs more than any of them. Consider next a deviation to $d_{2} \in\left(\max \left[102-2 b^{H}, 0\right], \max \left[102-b^{H}-b^{M}, 0\right]\right]$. Such a deviation produces at the same profiles as $d_{2}^{\prime}=\max \left[102-2 b^{H}, 0\right]$ and costs more than $d_{2}^{\prime}$. Consider a deviation to $d_{3} \in\left(\max \left[102-b^{H}-b^{M}, 0\right], 102-2 b^{M}\right)$. This produces at the same profiles as $d_{3}^{\prime}=\max \left[102-b^{H}-b^{M}, 0\right]$ and costs more than $d_{3}^{\prime}$. Consider a deviation to $d_{4} \in\left(102-2 b^{M}, 102-b^{L}-b^{H}\right)$. This produces at the same profiles as $d_{4}^{\prime}=102-2 b^{M}$ and costs more than $d_{4}^{\prime}$. Consider a deviation to $d_{5} \in\left(102-b^{L}-b^{H}\right.$, $\left.1022 b^{L} 2 b^{M}\right)$. This produces at the same profiles as $d_{5}^{\prime}=1022 b^{L} 2 b^{H}$ and costs more than $d_{5}{ }_{5}$. Consider a deviation to $d_{6} \in\left(1022 b^{L} 2 b^{M}, 102-2 b^{L}\right)$. This produces at the same profiles as $d_{6}^{\prime}=102-b^{L}-b^{M}$ and costs more than $d_{6}^{\prime}$. Finally, consider a deviation to $d_{7} \in\left(102-2 b^{L}, 102\right)$. This produces at the same profiles as $d_{7}^{\prime}=102-2 b^{L}$ and costs more than $d_{7}^{\prime}$.

Second, if $2 b^{M}<b^{L}+b^{H}$, consider $d_{3} \in\left(\max \left[1022 b^{H} 2 b^{M}, 0\right], \max \left[102-b^{H}-b^{L}, 0\right]\right)$ and $d_{3} \in\left(\max \left[102-b^{H}-b^{L}, 0\right], 102-2 b^{M}\right)$, rather than $d_{3} \in\left(\max \left[102-b^{H}-b^{M}, 0\right]\right.$, $\left.102-2 b^{M}\right)$ and $d_{4} \in\left(102-2 b^{M}, 102-b^{L}-b^{H}\right)$.

\section{References}

Andreoni, J., 1998. Toward a theory of charitable fundraising. Journal of Political Economy 106, 1186-1213.

Andreoni, J., 2001. The economics of philanthropy. In: Smeltser, N., Baltes, P. (Eds.), International Encyclopedia of Social and Behavioral Sciences. Elsevier, Oxford, pp. 11369-11376.

Bagnoli, M., McKee, M., 1991. Voluntary contributions games: efficient private provision of public goods. Economic Inquiry 29, 351-366.

Cadsby, Charles Bram, Maynes, Elizabeth, 1998. Voluntary provision of threshold public goods with continuous contributions: experimental evidence. Journal of Public Economics 71, 53-75.

Chen, Yan, 2003. An experimental study of serial and average cost pricing mechanisms. Journal of Public Economics 87, 235-255.

Chen, Yan, Khoroshilov, Yuri, 2003. Learning under limited information. Games and Economic Behavior $44,1-25$.

Croson, Rachel, Marks, Melanie, 1999. The effect of incomplete information in a threshold public goods experiment. Public Choice 99, 103-118.

Dawes, R., Orbell, J., Simmons, R., van de Kragt, A., 1986. Organizing groups for collective action. American Political Science Review 80, 1171-1185.

Deb, Rajat, Razzolini, Laura, 1999. Auction-like mechanisms for pricing excludable public goods. Journal of Economic Theory 88, 340-368.

Dorsey, Robert, Laura Razzolini, Michael Reksulak, 2002. An experimental evaluation of the serial cost sharing rule. Working Paper, University of Mississippi School of Business.

Ferejohn, John, Forsythe, Robert, Noll, Roger, 1979. An experimental analysis of decision making procedures for discrete public goods: a case study of a problem in institutional design. In: Smith, Vernon (Ed.), Research in Experimental Economics, vol. 1. JAI Press, Greenwich, CT.

Ferejohn, John, Forsythe, Robert, Noll, Roger, Palfrey, Thomas R., 1982. An experimental examination of auction mechanisms for discrete public goods. In: Kinder, Donald R., Palfrey, Thomas R. (Eds.), Experimental Foundations of Political Science, vol. 1993. University of Michigan Press, Ann Arbor, pp. 221-244.

Goeree, Jacob K., Holt, Charles A., Palfrey, Thomas R., 2002. Quantal response equilibrium and overbidding in private-value auctions. Journal of Economic Theory 104, 247-272.

Green, Jerry, Laffont, Jean-Jaques, 1977. Characterization of satisfactory mechanisms for the revelation of preferences for public projects. Econometrica 45, 427-438.

Isaac, R.M., Schmidtz, D., Walker, J., 1988. The assurance problem in a laboratory market. Public Choice 62, $217-236$. 
Ledyard, John, Palfrey, Thomas, 1999a. A characterization of interim efficiency with public goods. Econometrica $67,435-448$.

Ledyard, John, Palfrey, Thomas, 1999b. Interim efficiency in a public goods problem. In: d'Aspremont, C. (Ed.), Social Organization and Mechanism Design. De Boeck University Press, Brussels, pp. 239-261.

Ledyard, John, Palfrey, Thomas, 2001. The approximation of efficient public good mechanisms by simple voting schemes. Journal of Public Economics 83, 153-172.

McKelvey, Richard, Palfrey, Thomas, 1995. Quantal response equilibrium for normal form games. Games and Economic Behavior 7, 6-38.

McKelvey, Richard, Palfrey, Thomas, 1996. A statistical theory of equilibrium in games. Japanese Economic Review 47, 186-209.

McKelvey, Richard, Palfrey, Thomas, 1998. Quantal response equilibrium for extensive form games. Experimental Economics 1, 9-41.

Marks, Melanie, Croson, Rachel, 1998. Alternative rebate rules in the provision of a threshold public good: an experimental investigation. Journal of Public Economics 67, 195-220.

Marwell, G., Ames, R., 1979. Experiments on the provision of public goods: I. Resources, interest, group size, and the free-rider problem. American Journal of Sociology 84, 1335-1360.

Marwell, G., Ames, R., 1980. Experiments on the provision of public goods: II. Provision points, stakes, experience, and the free-rider problem. American Journal of Sociology 85, 926-937.

Moulin, Herve, 1994. Serial cost sharing of excludable public goods. Review of Economic Studies 61, $305-325$.

Moulin, Herve, Shenker, Scott, 1992. Serial cost sharing. Econometrica 60, 1009-1037.

Norman, Peter, 2000. Efficient mechanisms for public goods with use exclusions. Mimeo, University of Wisconsin Department of Economics.

Palfrey, Thomas R., Rosenthal, Howard, 1991. Testing game theoretic models of free riding: new evidence on probability bias and learning. In: Palfrey, Thomas (Ed.), Laboratory Research in Political Economy. University of Michigan Press, Ann Arbor.

Rapoport, A., 1985. Public goods and the MCS experimental paradigm. American Political Science Review, $148-155$.

Rapoport, A., 1987. Research paradigms and expected utility models for the provision of step-level public goods. Psychological Review 94, 74-83.

Rapoport, A., 1988. Provision of step-level public goods: effects of inequality in resources. Journal of Personality and Social Psychology 54, 432-440.

Rapoport, A., Eshed-Levy, D., 1989. Provision of step-level public goods: effects of greed and fear of being gypped. Organizational Behavior and Human Decision Processes 44, 325-344.

Rapoport, A., Suleiman, R., 1993. Incremental contribution in step-level public goods games with asymmetric players. Organizational Behavior and Human Decision Processes 55, 171-194.

Sidney, Siegel, 1956. Nonparametric Statistics for the Behavioral Sciences. McGraw-Hill, New York.

Smith, Vernon, 1979a. An experimental comparison of three public good decision mechanisms. Scandinavian Journal of Economics 81, 198-215.

Smith, Vernon, 1979b. Incentive compatible experimental processes for the provision of public goods. In: Smith, Vernon (Ed.), Research in Experimental Economics, vol. 1. JAI Press, Greenwich, CT, pp. 159-168.

Smith, Vernon, 1980. Experiments with a decentralized mechanism for public good decisions. American Economic Review 70, 584-599.

Suleiman, R., Rapoport, A., 1992. Provision of step-level public goods with continuous contribution. Journal of Behavioral Decision Making 5, 133-153.

van Dijk, Eric, Grodzka, Malgorzata, 1992. The influence of endowments asymmetry and information level on the contribution to a public step good. Journal of Economic Psychology 13, 329-342. 